Review Article

\title{
The Application of Traditional Chinese Medicine Injection on Patients with Acute Coronary Syndrome during the Perioperative Period of Percutaneous Coronary Intervention: A Systematic Review and Meta-Analysis of Randomized Controlled Trials
}

\author{
Zhaofeng Shi $\mathbb{D}$, ${ }^{1,2}$ Chen Zhao $\mathbb{D}^{3}{ }^{3}$ Jiayuan Hu, ${ }^{1,2}$ Qianqian Dai, ${ }^{1,2}$ Manke Guan, \\ Changming Zhong, ${ }^{1,2}$ Guihua Tian $\mathbb{1},{ }^{1,2}$ and Hongcai Shang $\oplus^{1,2,4}$ \\ ${ }^{1}$ Key Laboratory of Chinese Internal Medicine of Ministry of Education and Beijing, Beijing 100700, China \\ ${ }^{2}$ Dongzhimen Hospital, Beijing University of Chinese Medicine, Beijing 100700, China \\ ${ }^{3}$ Institute of Basic Research in Clinical Medicine, China Academy of Chinese Medical Sciences, Beijing 100700, China \\ ${ }^{4}$ International Evidence-Based Research Institute of Chinese Medicine, Beijing University of Chinese Medicine, \\ Beijing 100029, China
}

Correspondence should be addressed to Hongcai Shang; shanghongcai@foxmail.com

Received 4 November 2019; Revised 3 April 2020; Accepted 7 April 2020; Published 19 May 2020

Academic Editor: Ciara Hughes

Copyright ( $(2020$ Zhaofeng Shi et al. This is an open access article distributed under the Creative Commons Attribution License, which permits unrestricted use, distribution, and reproduction in any medium, provided the original work is properly cited.

Introduction. TCMI with the effect of Liqihuoxue and Yiqihuoxue has been applied as complementary therapies during the perioperative period of PCI for patients with ACS, while the recommended time points and plans of TCMI are still short of the support of evidence-based medicine. Methods. A systematic review and meta-analysis was conducted to evaluate the clinical efficacy and safety of TCMI on patients with ACS during the perioperative period of PCI. RCTs were searched based on standardized searching rules in seven medical databases from the inception up to August 2019. Two reviewers conducted the study selection, data extraction, and quality analysis independently. Data were analysed with the support of software RevMan and Stata. Results. A total of 68 articles with 6,043 patients were enrolled. The result of meta-analysis showed that the TCMI combined with western medicine was superior to the western medicine alone on clinical efficiency (before the PCI, before and after the PCI, or overall, $P<0.05$ ), the occurrence of MACE (myocardial infarction and stenocardia: before the PCI, before and after the PCI, or overall, $P<0.05$; arrhythmia: before and after the PCI, $P<0.05$ ), and the level of inflammatory factors (hs-CRP: before the PCI, before and after the PCI, or overall, $P<0.05$; IL-6: after the PCI, $P<0.05$ ). The TCMI with the effect of Liqihuoxue obtained more support compared with Yiqihuoxue based on the result of meta-analysis. Conclusions. TCMI with the effect of Liqihuoxue or Yiqihuoxue combined with western medicine generally showed the potential advantage on the treatment of ACS during the perioperative period of PCI. However, the optimal time point of intervention and recommended plan based on the effect still needs more clinical evidence. We consider that the research of precise and standardized application of TCMI will be a promising direction for TCM in the future.

\section{Introduction}

Acute coronary syndrome (ACS), which is caused by rupture or erosion of atherosclerotic plaque in the coronary artery or fresh thrombosis, can be classified as unstable angina (UA), non-ST-elevation myocardial infarction (NSTEMI), and STelevation myocardial infarction (STEMI) based on the electrocardiographic changes and cardiac biomarker [1]. In most developed countries, the incidence of ACS is declining in the past 30 years $[2,3]$; however, it is still increasing in China with each passing year and the vast majority of patients with ACS was first diagnosed and received treatment in the emergency department [4]. There are currently 290 million cardiovascular patients in China, and the number of 
patients with ACS is expected to reach 22.6 million by 2030 [5].

The clinical manifestation of ACS patients is variable, with the most common symptom such as chest pain or chest tightness [6]. However, some patients such as elderly women and diabetes may not have typical symptoms. The diagnosis of ACS can be defined as the increase in troponin levels with at least one value $>$ 99th percentile of upper reference limit and plus the at least one part of diagnostic evidence from the symptom of myocardial ischemia, electrocardiograph (ECG), and image finding [7]. The risk stratification for ACS is a prerequisite on the establishment of clinical strategy, which means only by applying an appropriate risk stratification, a preferable therapeutic efficiency can be achieved. Some publications have identified new biomarkers for risk stratification of patients with ACS, including gut-microbiota-dependent trimethylamine $\mathrm{N}$-oxide [8], microRNAs (26b-5, 660-5, and 320a) [9], and acute myocardial infarction (AMI) telomere length in peripheral blood cells [10]. As for the clinical score for risk stratification, the PRECISE-DAPT (dual antiplatelet therapy) [11] and the CRUSADE bleeding score [12] has proved its value on the prediction of the risk of bleeding events; meanwhile, the Global Registry of Acute Coronary Events (GRACE) score and the thrombolysis in myocardial infarction (TIMI) score have identified the effect on the evaluation of ischemia risk [13]. Basic treatments for ACS include dual antiplatelet (such as aspirin and P2Y12 inhibitors) [14], anticoagulant (such as fondaparinux and low-molecular-weight heparin) [15], and anti-ischemic (such as beta-blockers) [16] therapies. The treatment of revascularization includes the percutaneous coronary intervention (PCI), thrombolytic therapy (tissue plasminogen activator), and coronary artery bypass grafting (CABG) [17].

PCI, which owns the immediate effect on revascularizing the infarct-related arteries (IRA), is being widely applied and dramatically improved the prognosis of ACS [18]. In 2015, more than 567,000 patients registered and finished the PCI in China, ranking the second in the world [19]. It should be noticed that this figure reached 753,142 in 2017 based on the report of China Cardiovascular Intervention Forum (CCIF). However, despite the improvement in antithrombotic technology and innovation of revascularizing strategy, the prognosis of PCI for patients with ACS is still unsatisfactory [20], and the incidence of major adverse cardiac events (MACE) is still at a high level [21]. Some PCI-related problems, such as no-reflow, ischemia-reperfusion injury, perioperative myocardial injury (PMI), in-stent restenosis, and stent thrombosis, are difficult to avoid. In the past 30 years, with the development in clinical trials of TCM in China, it has been found that the traditional Chinese medicine injection (TCMI) has a good effect on treating and preventing arrhythmia and reperfusion injury, improving heart function and protecting myocardium [22]. The Liqihuoxue and Yiqihuoxue are two essential effects of TCMI. According to the theory of TCM, Qi is the most basic substance to constitute and maintain human life activities. The stagnation or deficiency of $Q i$ will induce the blood stasis, which is basically equivalent to endothelial dysfunction (ETDF), forming an essential pathological basis of cardiovascular disease. Liqihuoxue is used in the ACS patients with asthenia syndrome through the function of regulating $Q i$ and removing blood stasis, while Yiqihuoxue is used for the deficiency syndrome through the function of nourishing $Q i$ and removing blood stasis.

The application of TCMI combined with western medicine during the perioperative period of $\mathrm{PCI}$ has become a hotspot on the treatment of ACS in China, but the optimal time point of intervention is still a matter of debate and the recommended plan from TCMI with the effect of Liqihuoxue or Yiqihuoxue is still unknown. Moreover, some clinical centers randomly use the TCMI with the effect of Liqihuoxue and Yiqihuoxue before or even after PCI. Finding the optimal time point of intervention and providing the therapeutic plan based on the effect of Chinese medicine are necessary for the development of TCM. Given the great variation in previous results, we performed a systematic review and meta-analysis to evaluate the efficacy and safety of TCMI in the treatment of ACS based on the different time points and the effect of Liqihuoxue or Yiqihuoxue.

\section{Methods}

This research is based on the guideline of PRISMA [23] and followed the instruction from the Cochrane Reviewer Handbook (version 5.1) [24].

2.1. Data Sources and Search Methods. Seven electronic medical databases named PubMed, Cochrane Library, Web of Science, EMBASE, the CNKI (Chinese), Wanfang Data (Chinese), and Vip Data (Chinese) were searched from the inception up to August 2019. Articles were included with the language of Chinese or English. The relevant systematic reviews were also temporarily included and analysed for the supplementation of the potentially qualified articles. Emails were sent to authors for the acquirement of the non-full-text articles. The supplemental search was performed in the library of Beijing University of Chinese Medicine and the China Academy of Traditional Chinese Medicine for the acquisition of grey studies. The searching terms, which were conducted and adjusted for the variation in language, contained as follows: acute coronary syndrome, myocardial infarction, acute myocardial infarction, ST-segment elevation myocardial infarction, non-ST-segment elevation myocardial infarction, STEMI, NSTEMI, unstable angina, UA, injection, Chinese patent medicine, traditional Chinese medicine, percutaneous coronary intervention, PCI, and randomized clinical trials.

2.2. Eligibility Criteria. The eligibility criteria of inclusion and exclusion were performed by two researchers (MD. Zhaofeng Shi and MM. Qianqian Dai) independently, and the disagreement was resolved by the common discussion or the guidance of the third researcher (Pro. Hongcai Shang).

The eligibility criteria of included studies were suited for the following criteria: (1) RCTs; (2) patients who complied with the diagnostic criteria of ACS based on the guideline of 
ESC for STEMI [25] or UA/NSTEMI [26]; (3) patients of either gender and of any age who received the PCI, including the PTCA and coronary artery stent implantation (such as bare metal stent and drug eluting stent), within 12 hours from the occurrence of symptoms of myocardial ischemia; (4) patients who received the TCMI with the effect of regulating $Q i$ and removing stasis (Liqihuoxue) or nourishing $Q i$ and removing stasis (Yiqihuoxue) based on the guidelines of drug description. TCMI combined with western medicine (dual antiplatelet, anticoagulant, and antiischemic) was defined as the experimental group; meanwhile, western medicine alone was as the controlled group; (5) the time point of intervention for TCMI was settled before the PCI (less than 3 hours), after the PCI (more than 3 hours), or before and after the PCI together; (6) the outcome indicators should include at least one of following items: (a) clinical efficiency (including the criteria of complete response, partial response, and invalid response; complete response plus partial response was defined as the total effective response) [27]; (b) MACE (including death, myocardial infarction, hospitalization for unstable angina, transient ischemic attack and stroke, heart failure event, percutaneous coronary intervention, peripheral vascular intervention, and stent thrombosis) [28]; (c) inflammatory factors (CRP, hs-CRP, IL-6, IL-10, IL-18, or TNF- $\alpha$ ); (d) adverse events resulting from TCMI or western medicine.

Studies were excluded if they met one of the following criteria: (1) non-RCTs (including quasi-RCTs, CCTs, cohort study, case series, and case reports); (2) received the traditional Chinese herbal medicine or TCMI in the controlled group; (3) received the unrelated TCMI, which was not focused on the treatment of ACS, or Chinese herbal medicine in the experimental group; (4) the types of diseases were not compatible with the criteria of ACS (STEMI, USTEMI, and UA); and (5) severe clinical illness, including (a) had active bleeding or the tendency of bleeding; (b) cardiogenic shock, cardiac rupture, or ventricular septal perforation; (c) acute pericarditis, subacute infective endocarditis, or aortic dissection; (d) severe arrhythmia (left bundle branch block, ventricular tachycardia, ventricular flutter, and ventricular fibrillation); and (e) serious disease in the liver, kidney, hematopoietic system, or malignant tumours.

Particularly, it should be highlighted that STEMI, NSTEMI, and UA had many commonalities in the pathogenesis and pathophysiology, which were related to the formation of atherosclerotic plaque. Although the difference among them was the degree of occlusion of coronary artery (STEMI is more seriously than NSTEMI), the long-term prognosis and the severity were similar and the treatment of PCI was of great significance. As for the classifications of stents in the insertion of vessel stents, even though the BVS (bioresorbable vessel scaffold) was no worse than EES (everolimus-eluting stent) in 1-year TLF (target lesion failure) rate, cardiogenic death, and TLR (target lesion revascularization) induced by target vessel MI and ischemia [29], we did not limit the type of stent in the inclusion criteria of this research in view of the current status of PCI in China. Chinese herbal medicine should not be combined with TCMI, even though they had the synergistic effects without interfering with the major function of TCMI. The dosage of the TCMI and western medicine was discrepant in experimental groups or controlled groups, and there was no limitation for the dosage in the selection of research.

2.3. Study Selection. The software named EndNote X8 was used to establish a preliminary literature database which met the requirements of removing duplicates and screening the procedure of selection. Two researchers (MD. Zhaofeng Shi and Prof. Chen Zhao) did the procedure by reading title and abstract based on the previously defined inclusion and exclusion criteria. After obtaining the full-text papers, the researchers read the inclusion and exclusion criteria once again for further screening. If the information of the included papers was incomplete or difficult to be judged during the process of screening, the original author would be contacted by email. If it was difficult to receive a response from the original author, the missing information would be excluded. The third researcher (Prof. Hongcai Shang) did the judgment after the discussion if there was disagreement during the cross-correction.

2.4. Data Extraction and Quality Analysis. Two researchers (MM. Changming Zhong and MD. Zhaofeng Shi) extracted data and established a summary table independently, which contained the following items: (1) the name of author and the year of publication, (2) the researching area, (3) sample size, (4) age of patients, (5) other information (such as the past medical history, personal history, and classification of heart function), (6) treatments of experimental and controlled groups, (7) duration of treatments and follow-up, (8) evaluation of outcome indicators and quality assessment, and (9) adverse events of the TCMI. The results were crosschecked in this process, and any disagreement between the results will be resolved after a discussion and judged by the arbiter (Prof. Hongcai Shang).

The quality analysis was performed by two investigators independently (MD. Zhaofeng Shi and MD. Jiayuan $\mathrm{Hu}$ ), using the tool of the Cochrane Reviewer Handbook 5.1 [24]. This tool was conducted to evaluate the risk bias of included studies across seven domains: (1) random sequence generation (selection bias), (2) allocation concealment (selection bias), (3) blinding of participants and personnel (performance bias), (4) blinding of outcome assessment (detection bias), (5) incomplete outcome data (attrition bias), (6) selective reporting (reporting bias), and (7) other sources of bias (other bias). Researchers would answer these questions with "yes (Y)," "unclear (U)," or "no $(\mathrm{N})$ " to evaluate the degree of risk of bias. If an included research is satisfied with more than four domains, it should be grouped as the low risk of bias; one to four domains should be grouped as the moderate risk of bias; and one or no domain should be grouped as the high risk of bias. The disagreement during this procedure would be resolved after a discussion and judged by the arbiter (Prof. Hongcai Shang). The outcomes above were established as tables and images with the support of software Review Manager (RevMan, version 5.3, the 
Nordic Cochrane Centre, the Cochrane Collaboration, 2012 Copenhagen, Denmark).

2.5. Statistical Analysis. The data were analysed by the software RevMan and Stata (version 14.0, StataCorp LP, College Station, US). The analysis was conducted after the comparison of outcomes between the experimental and the controlled groups. The risk ratio (RR) with 95\% confidence interval (CI) was calculated for the dichotomous data and the standard mean difference (Std. $\mathrm{MD}$ ) or the mean difference (MD) with $95 \%$ CI was calculated for the continuous data, respectively.

The $\chi^{2}$ test and the $I^{2}$ statistic were conducted to identify and measure the statistical heterogeneity. These methods could provide an estimate of variation which resulted from heterogeneity. The heterogeneity was divided into three levels based on the $I^{2}$ statistic outcomes: (1) between 25 and $50 \%$ was low, (2) between 50 and $75 \%$ was moderate, and (3) above $75 \%$ was high. The $P$ value lower than 0.05 and $I^{2}$ statistic outcome higher than $50 \%$ were considered to obtain significant heterogeneity. The heterogeneity source needed to be further explored with the method of subgroup analysis or metaregression analysis. The sample size, research areas, and levels of hospitals were used as the classification for subgroup analysis.

A random-effects model which used the method of DerSimonian-Laird (DS-L) [30] or Inverse Variance (IV) was conducted to pool data based on the moderate or high heterogeneity and a fixed-effects model which used the method of Mantel-Haenszel (M-H) was established to pool data based on the low heterogeneity [31]. The sensitivity analysis was conducted to evaluate the stability of analysis by using different effects model and examining the effects of individual factors on the overall combined effect size. The method of funnel plot and Egger's test/Begg's test was used to assess the publication bias by the software RevMan and Stata if an outcome included more than 10 studies [32, 33].

\section{Results}

3.1. Study Selection. The flow diagram of the screening and selection of potential articles was illustrated in Figure 1. A total of 579 related studies were identified from the medical databases, and 342 studies were ruled out due to the duplication. After the screening of the title and abstract, one hundred and forty-two studies were further excluded for the following reasons: (1) twenty-eight were experimental studies, (2) sixty-six clinical studies did not belong to RCTs, (3) fifteen studies belonged to reviews or meta-analyses, (4) twenty-two studies were protocols, and (5) eleven studies could not obtain the full-text paper. There were 27 studies excluded after the full-text paper reading for the following reasons: (1) the experimental group was not eligible for 6 studies, (2) the controlled group was not eligible for 2 studies, (3) insufficient data were found in 7 studies, and (4) twelve studies had inappropriate criteria for the indicators of outcome. Overall, a total of 68 articles with 6,043 patients were enrolled in this research.
3.2. Study Characteristics. A total of 68 studies conformed to the final eligibility criteria and were included in the metaanalysis (Table 1). All studies were randomized clinical trials (RCTs) and fifteen trials among them were multicentred studies, which performed in different hospitals of China [34, $48,49,51,55,58,59,61,66,82,88,94,95,100,101]$. The publishing year of studies was found between 2004 and 2018. The sample size of studies ranged from 38 [46] to 203 [65], and the age range of male and female was between 31 [37] and 84 [41] years old. As for the classification of ACS, only twenty-one studies clearly defined including seven studies for UA [36, 73, 74, 78, 80, 86], eleven studies for STEMI [42, $45,46,49,51,66,70,71,91,92,95]$, and three for NSTEMI $[52,72,75]$. However, the rest of forty-eight studies did not introduce the classification. The types of TCMI in the experimental group were diversified and listed as follows: injection of Dazhuhongjingtian [34-38], Shuxuetong [39, 42, 83-89], Shenmai [40-44, 46-48], Danshen [45, 49], Danhong [50-67, 73, 74], Dengzhanhuasu [68], Gualoupi [69], Guanxinning [70, 71], Safflower yellow [72, 75], Safflower [76-78], Kudiezi [79], Shengmai [80-82], Xiangdan [90], Xuesaitong [91-95], Xueshuantong [96-100], and Yiqifumai [101]. The detailed information of TCMI, which included constituents of TCMI, Latin names of constituents for Chinese medicine, ratios of constituents, specifications clinical use of the TCMI, and Chinese national medicine permission numbers, was well illustrated (see Table S2 and Figures S13-S28 in the Supplementary Materials). The western medicine contained the anticoagulant, antimyocardial ischemia, antiplatelet, lipid-lowering, and antihypertensive treatment. As for the duration of therapy, all included studies except seven $[56,71,72,78,80,81,100]$ clearly reported. The time of follow-up was mentioned in fifteen included studies $[43,44,46,48,51,53,58,71,75,76$, $79,95,97,99,100]$. It needs to highlight that only fourteen included studies $[37,41-43,46,50,51,57,59,60,62,91,92$, 95] reported the adverse events, which focused on the bleeding event, gastrointestinal reaction, and arrhythmia.

3.3. Quality Analysis. For the included studies, twenty-two $[42,47,50,51,54,55,57,60,62,63,72,73,76,81,83-85,91$, $92,96,97,100]$ mentioned the random sequence generation. No study clearly illustrated or contained the allocation concealment. Only 2 studies $[74,76]$ introduced the blinding method, which was the sealed envelope method. As for the aspect of incomplete outcome data, no included studies had the attrition bias basically. Only 6 studies [48, 78, 87-90] had the question of existing of other biases (see Figures S1 and S2 and Table S1 in the Supplementary Materials).

\subsection{Meta-Analysis}

3.4.1. Clinical Efficiency. Figure 2 illustrates the clinical efficiency of TCMI based on the effect of Yiqihuoxue or Liqihuoxue and the time points of intervention. There were 15 articles including 3,332 participants analysed in the forest plot $[34,35,40,41,51-53,59,65,74,75,77,83,87$, 90]. We extracted 8 articles [34, 35, 41, 51, 52, 59, 65, 87] 


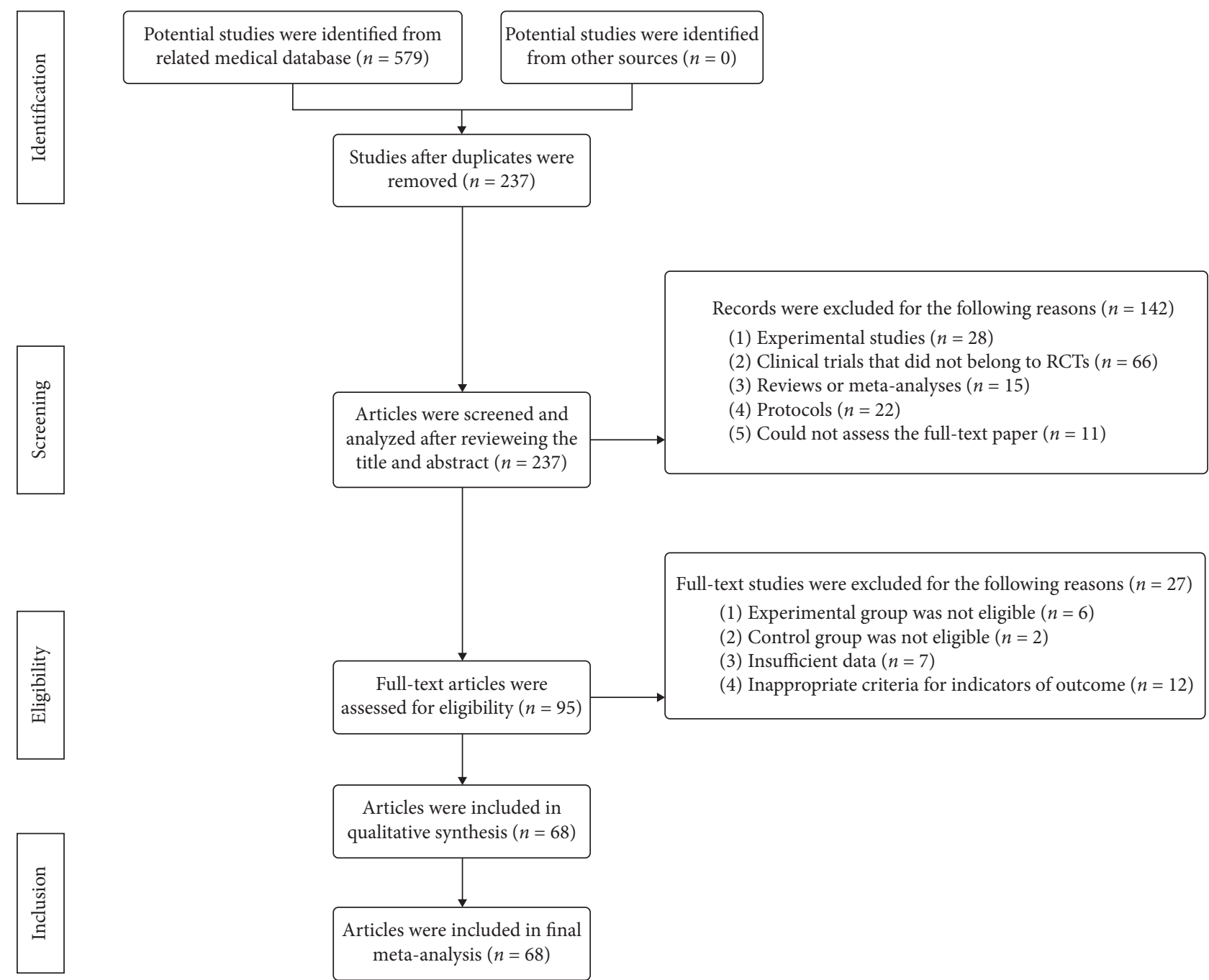

FIGURE 1: The preferred reporting items for systematic reviews and meta-analyses (PRISMA) flow diagram.

(2,090 participants) from the 15 studies to compare with the rest of 7 articles [40, 53, 74, 75, 77, 83, 90] $(1,242$ patients) based on the different time points of intervention during the perioperative period of PCI. The result showed that the clinical efficiency of TCMI combined with the western medicine (experimental group) was superior to the western medicine alone (controlled group) on patients with ACS (before the PCI: RR $=1.15,95 \% \mathrm{CI}=1.10$ to 1.20 , $P<0.01$; before and after PCI: $\mathrm{RR}=1.24,95 \% \mathrm{CI}=1.16$ to 1.34, $P<0.01$; overall: $\mathrm{RR}=1.18,95 \% \mathrm{CI}=1.14$ to 1.23 , $P<0.01)$. The TCMI with the effect of Liqihuoxue $[34,35$, $51-53,59,65,74,75,77,83,87]$ combined with western medicine was superior to the western medicine in the time points of before and after the PCI and after the PCI. The results of the clinical efficiency between the experimental group and the controlled group had statistical difference. The heterogeneity was small (before the PCI: $P=0.33$, $I^{2}=12 \%$; before and after the PCI: $P=0.79, I^{2}=0 \%$; overall: $P=0.13, I^{2}=13 \%$ ), and the fixed-effects model was performed to calculate combined data by the $M-H$ test. However, the results could not recommend the best time point of intervention for TCMI on ACS.
3.4.2. MACE. Figures 3-6 illustrate the MACE of patients with ACS after the treatment of experimental group and controlled group based on the effect of Liqihuoxue or Yiqihuoxue and the time point of intervention.

(1) All-Cause Mortality. There were 6 articles including 508 participants analysed the all-cause mortality in the forest plot $[49,57,71,76,83,84]$ (Figure 3). Three articles $[49,83,84]$ with 250 participants received the treatment before and after the PCI compared with the rest of 3 articles [57, 71, 76] with 258 patients received the treatment after the PCI. The meta-analysis showed that the occurrence of all-cause mortality of the experimental group after the PCI, before and after the PCI, and overall was not lower than the controlled group on patients with ACS (before and after the PCI: $\mathrm{RR}=0.71,95 \% \mathrm{CI}=0.23$ to 2.18, $P=0.55$; after the PCI: $\mathrm{RR}=0.66,95 \% \mathrm{CI}=0.23$ to 1.85, $P=0.42$; overall: $\mathrm{RR}=0.68,95 \% \mathrm{CI}=0.32$ to 1.46 , $P=0.32$ ). TCMI with the effect of Liqihuoxue or Yiqihuoxue $[57,76,83,84]$ did not show the superiority. The heterogeneity was not found (before and after the PCI: $P=0.44, I^{2}=0 \%$; after the PCI: $P=0.89, I^{2}=0 \%$; overall: 


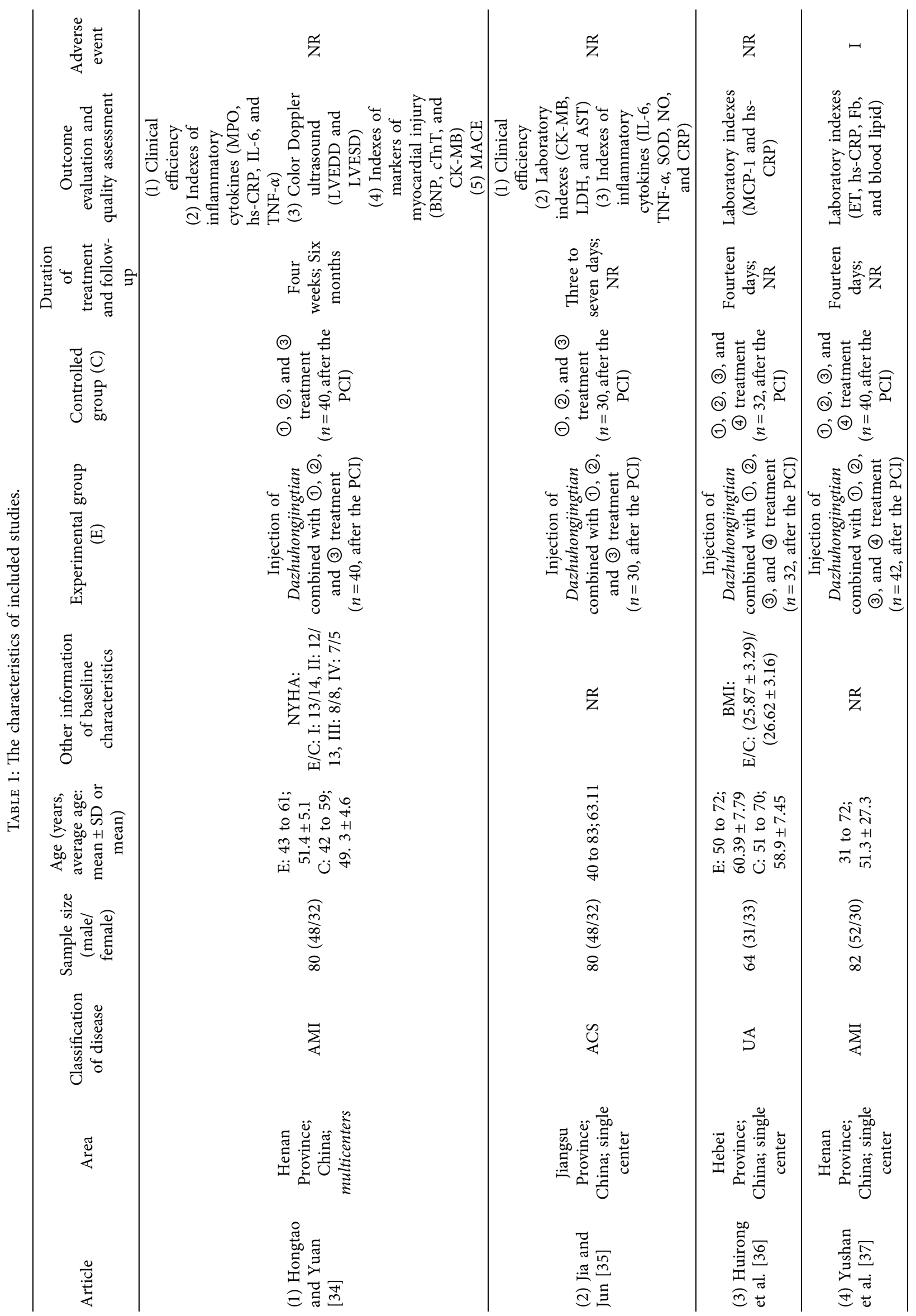




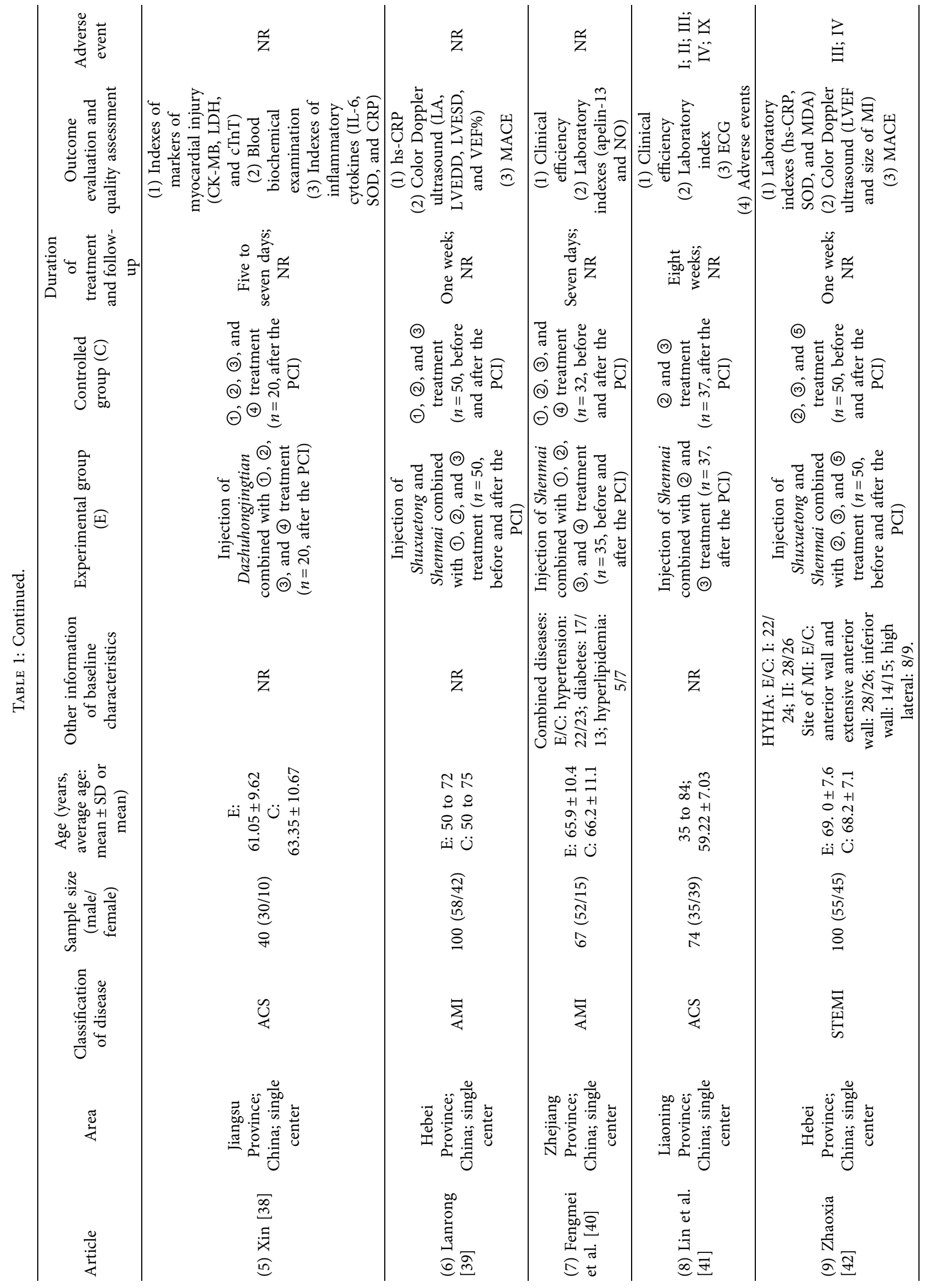




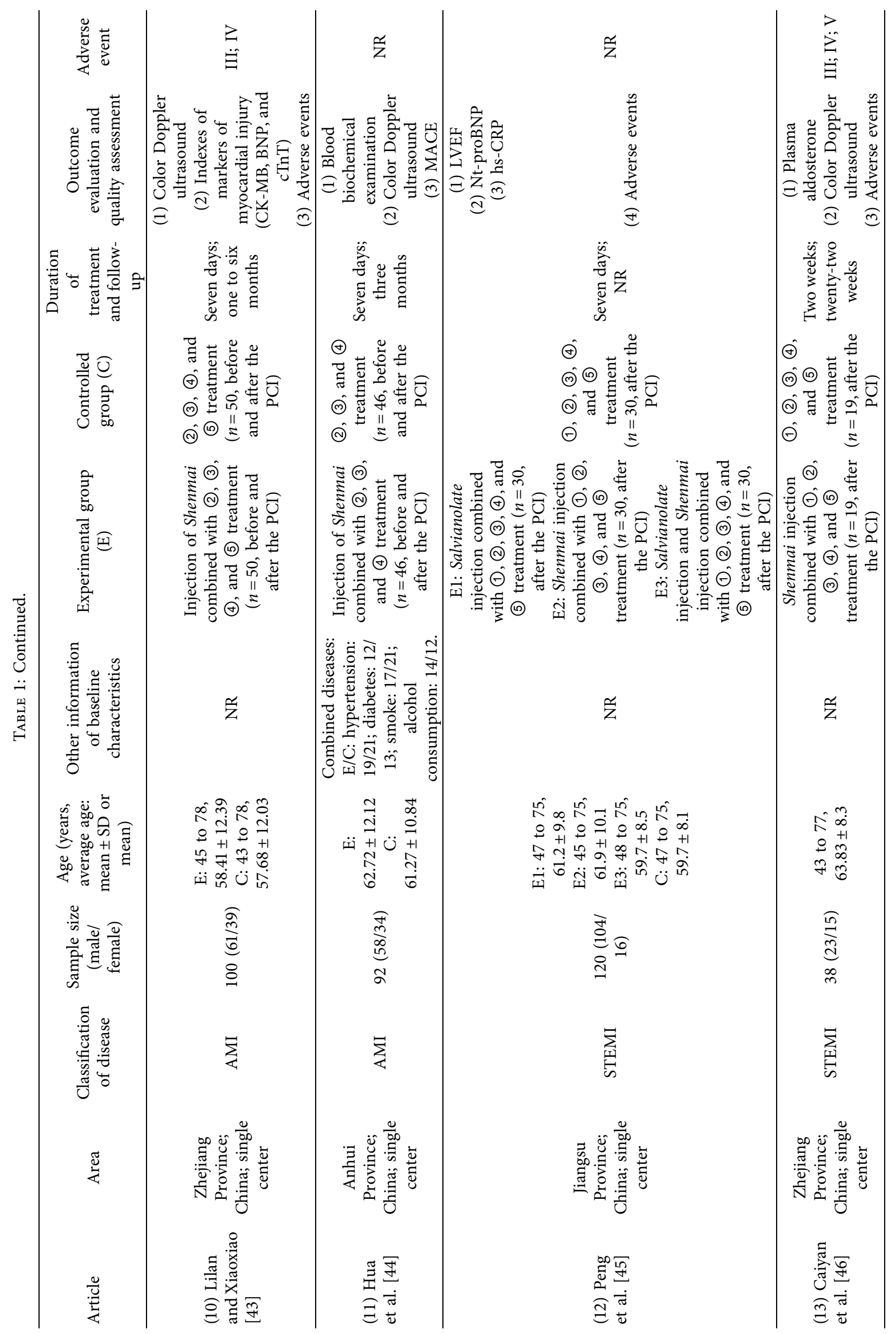




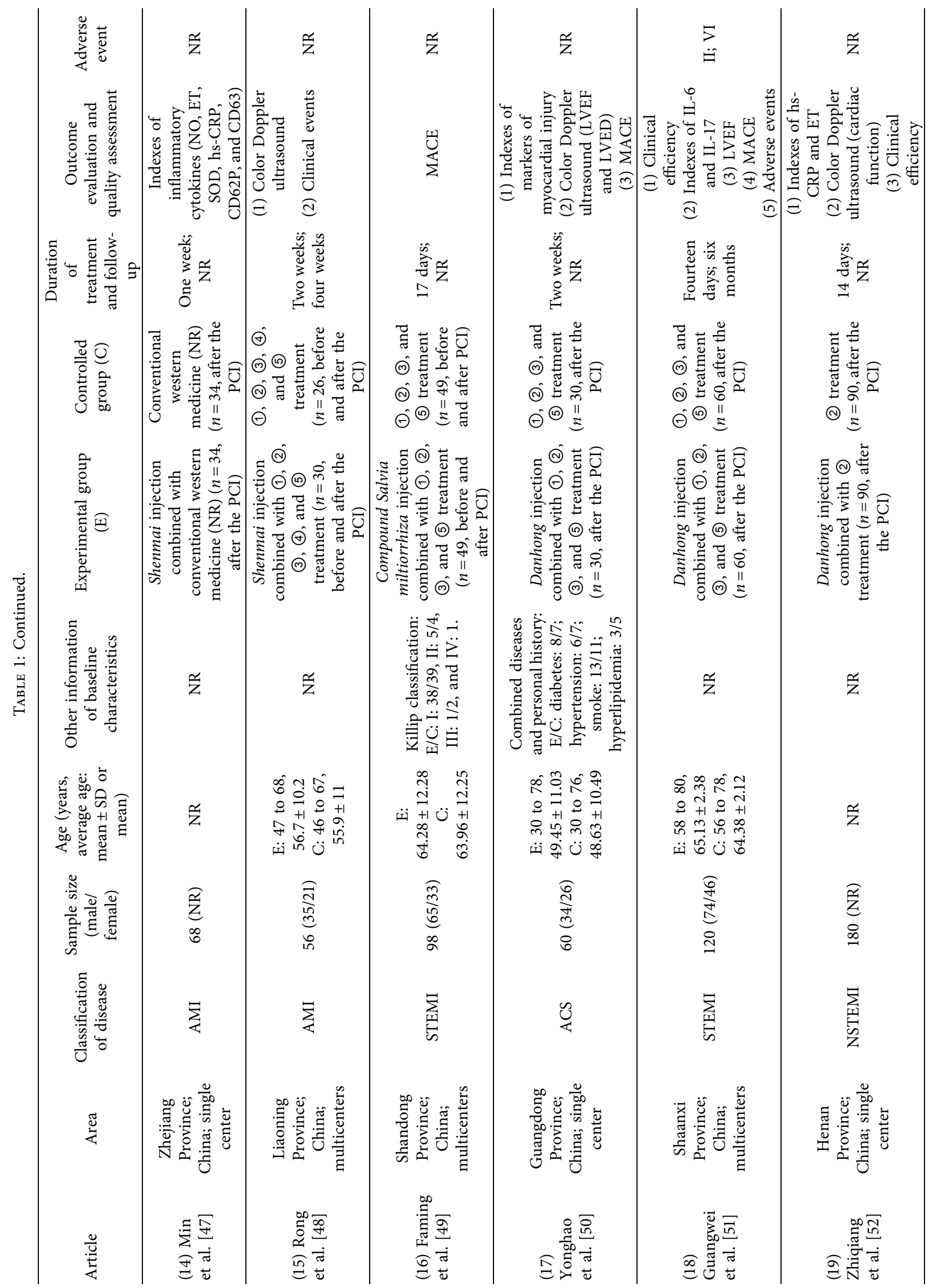




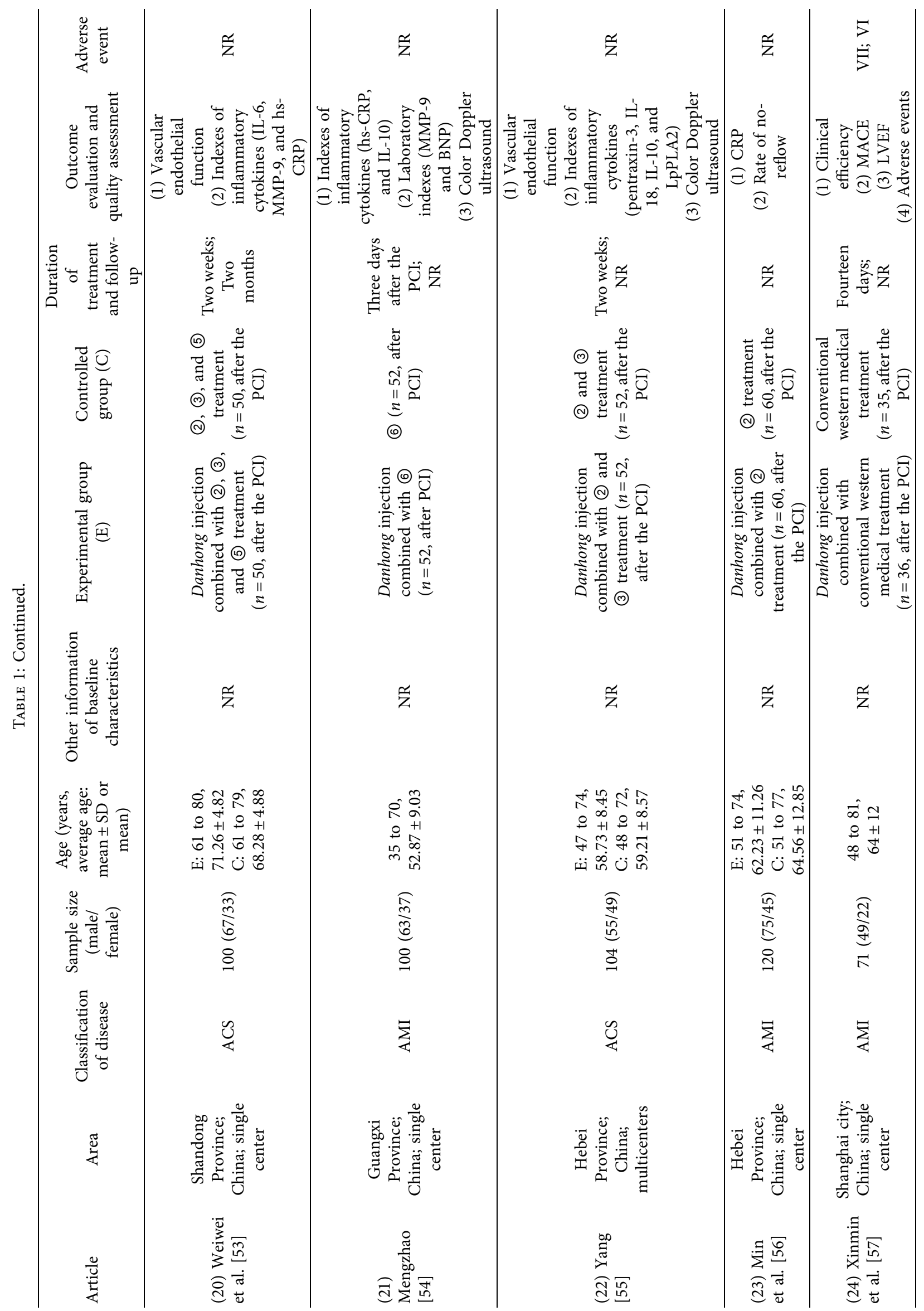




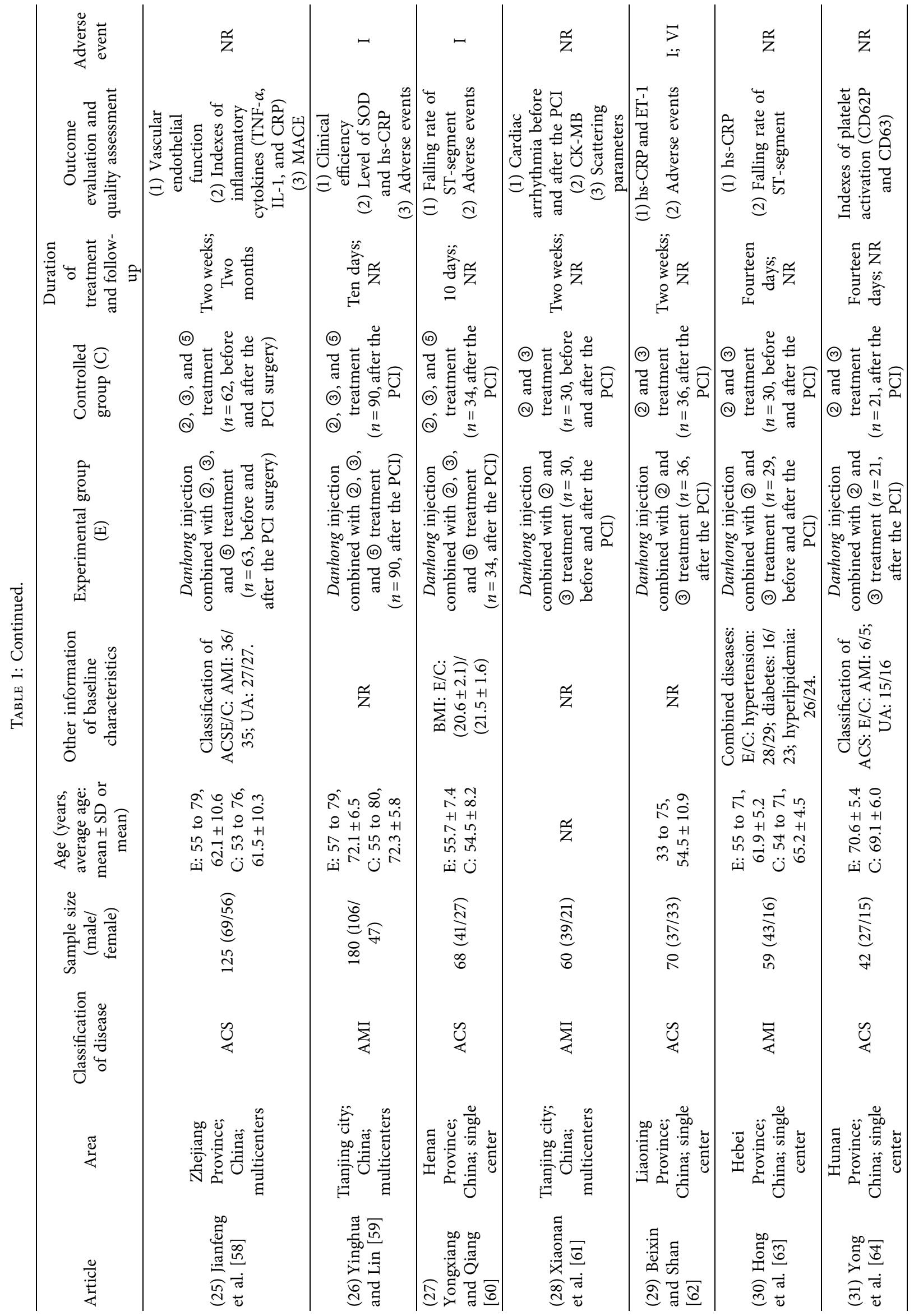




\begin{tabular}{|c|c|c|c|c|c|c|c|}
\hline 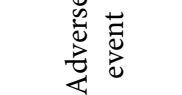 & 孚 & 孚 & 岂 & 孚 & 光 & 孚 & 色 \\
\hline 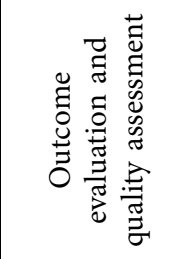 & 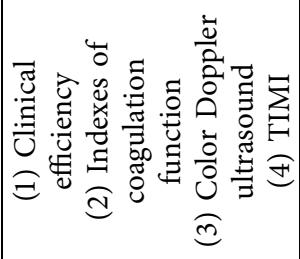 & 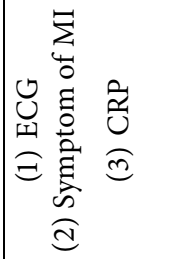 & 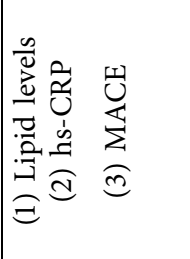 & 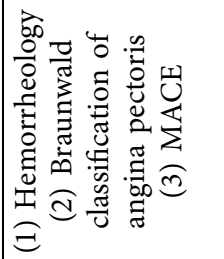 & 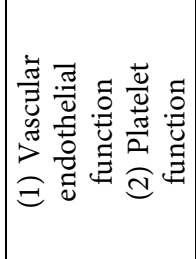 & 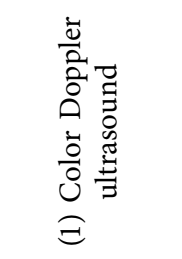 & 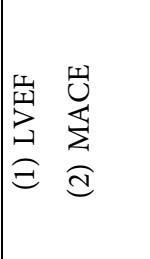 \\
\hline 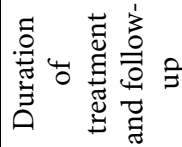 & 离 & 节商号 & 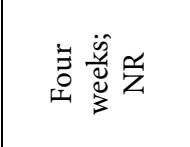 & 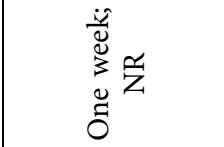 & 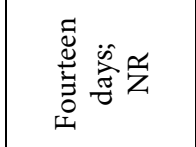 & 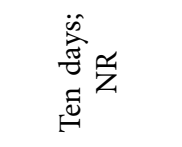 & 茫总总 \\
\hline 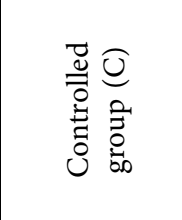 & 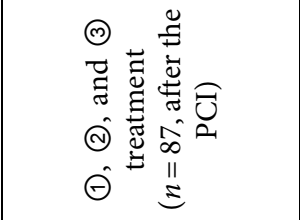 & 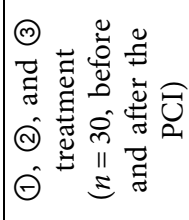 & 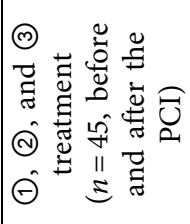 & 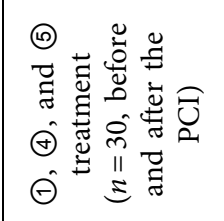 & 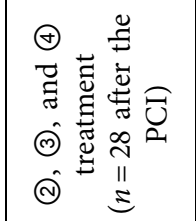 & 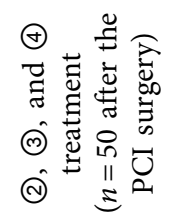 & 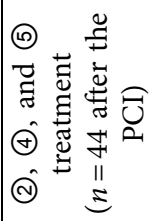 \\
\hline 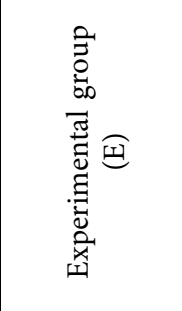 & 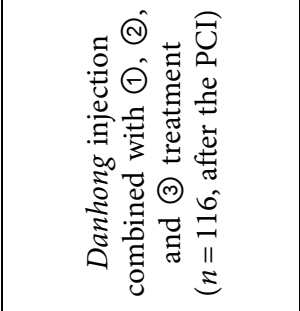 & 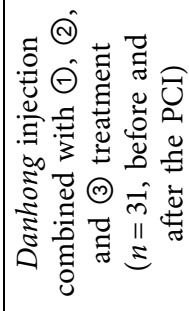 & 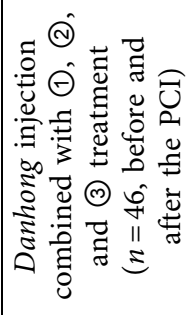 & 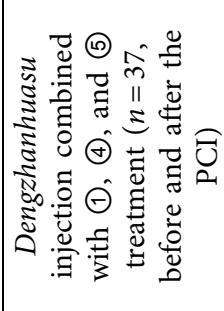 & 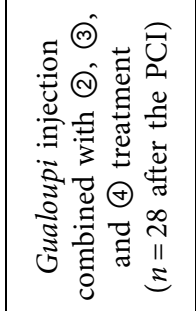 & 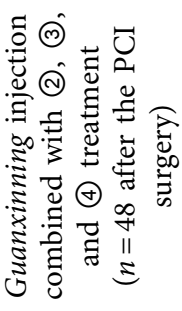 & 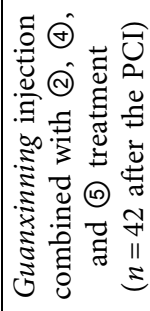 \\
\hline 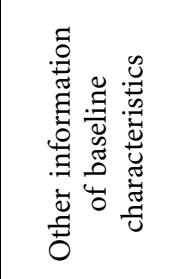 & 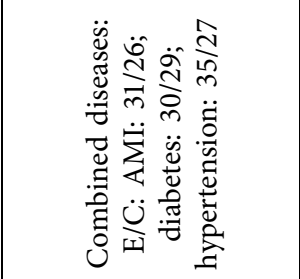 & 㟔 & 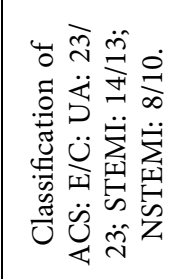 & 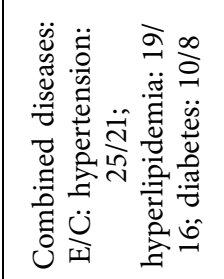 & 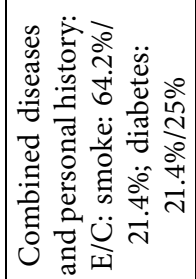 & 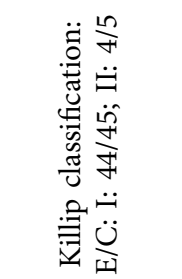 & 号 \\
\hline 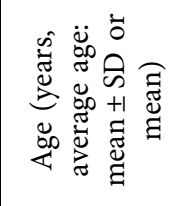 & 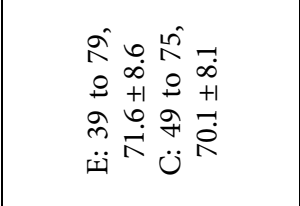 & 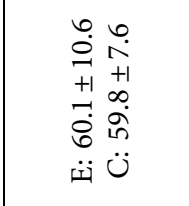 & 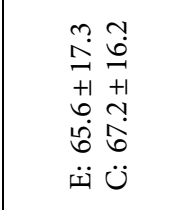 & 孚 & $\begin{array}{ll} & 0 \\
0 & 0 \\
+1 & +1 \\
0 & +1 \\
0 & 0 \\
0 & 0 \\
0 & 0 \\
4 & 0\end{array}$ & 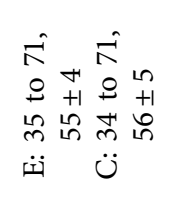 & $\begin{array}{l}\mathbb{N} \\
\stackrel{0}{0} \\
\stackrel{m}{m}\end{array}$ \\
\hline 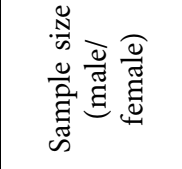 & 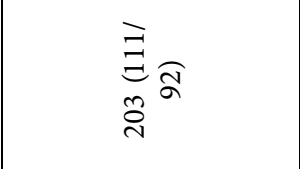 & $\begin{array}{l}\underset{\widetilde{N}}{\infty} \\
\infty \\
\stackrel{\infty}{\sigma} \\
\overrightarrow{0}\end{array}$ & 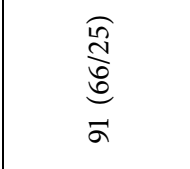 & $\begin{array}{l}\text { 孚 } \\
\text { 它 }\end{array}$ & 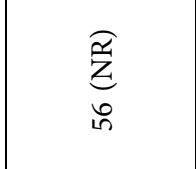 & 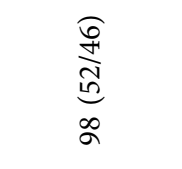 & 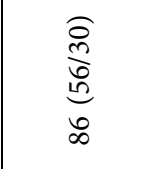 \\
\hline 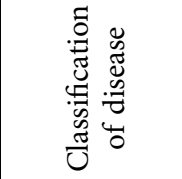 & $\sum_{4}$ & $\sum_{i=1}^{E}$ & $\mathcal{Z}_{4}$ & $\tilde{U}$ & $\tilde{Z}_{4}$ & $\underset{\substack{M \\
\text { 至 }}}{E}$ & 主 \\
\hline 蛋 & 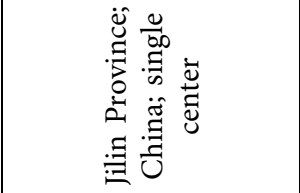 & 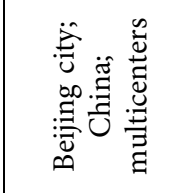 & 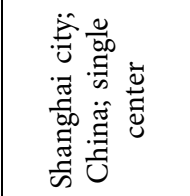 & 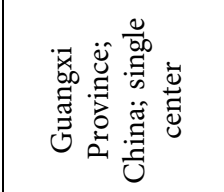 & 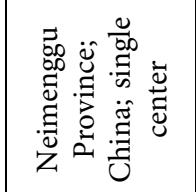 & 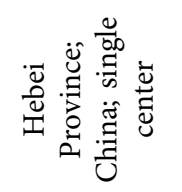 & 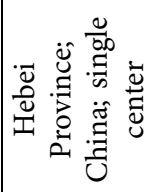 \\
\hline$\overline{\bar{Z}}$ & 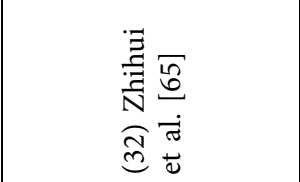 & 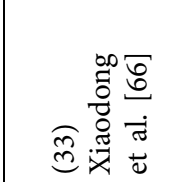 & 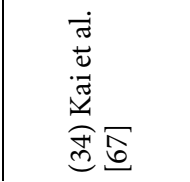 & 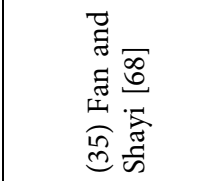 & 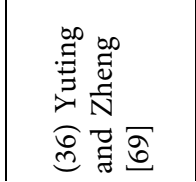 & 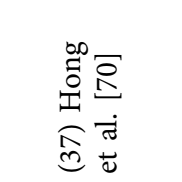 & 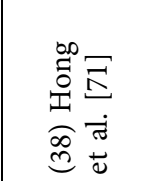 \\
\hline
\end{tabular}




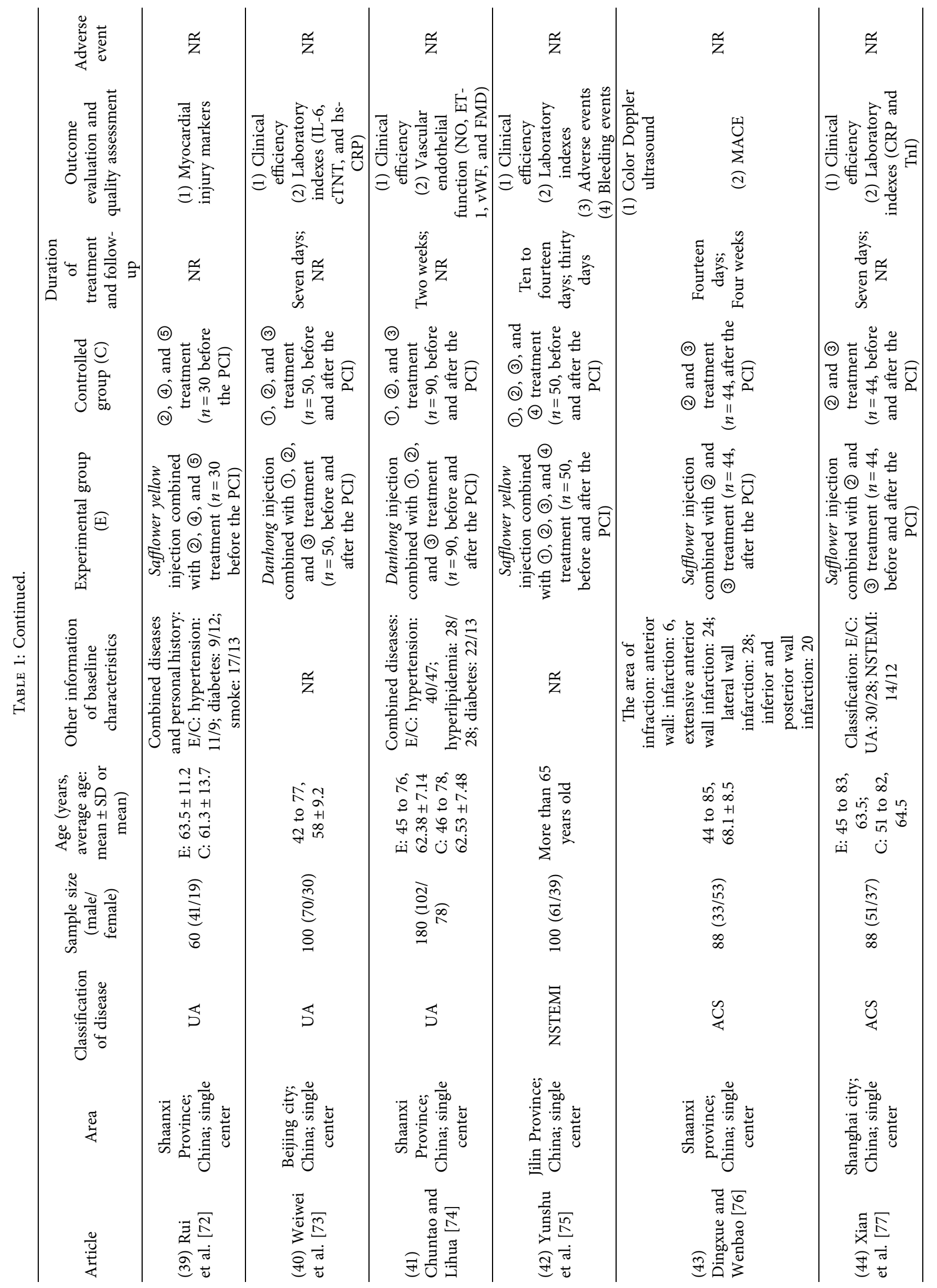




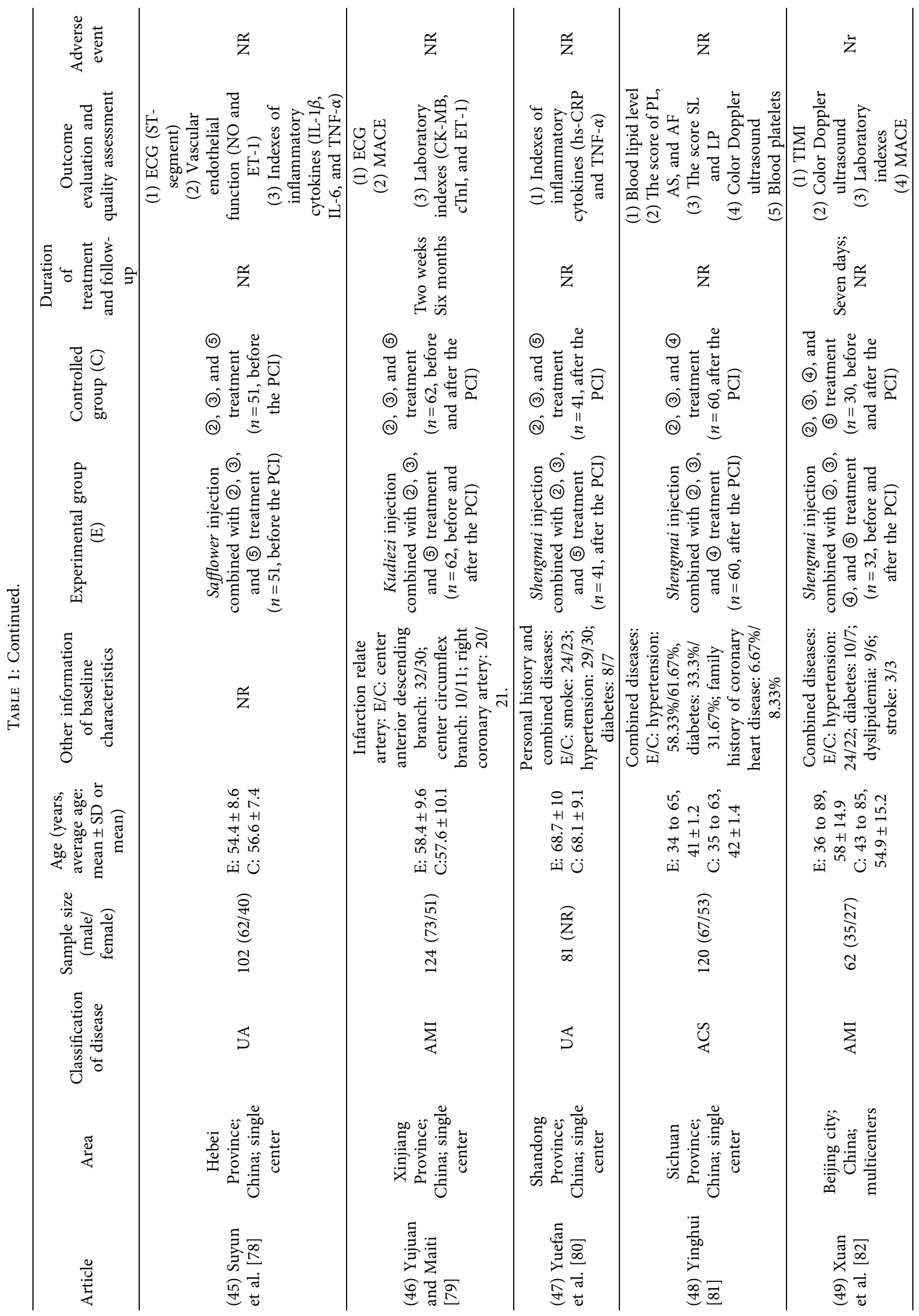




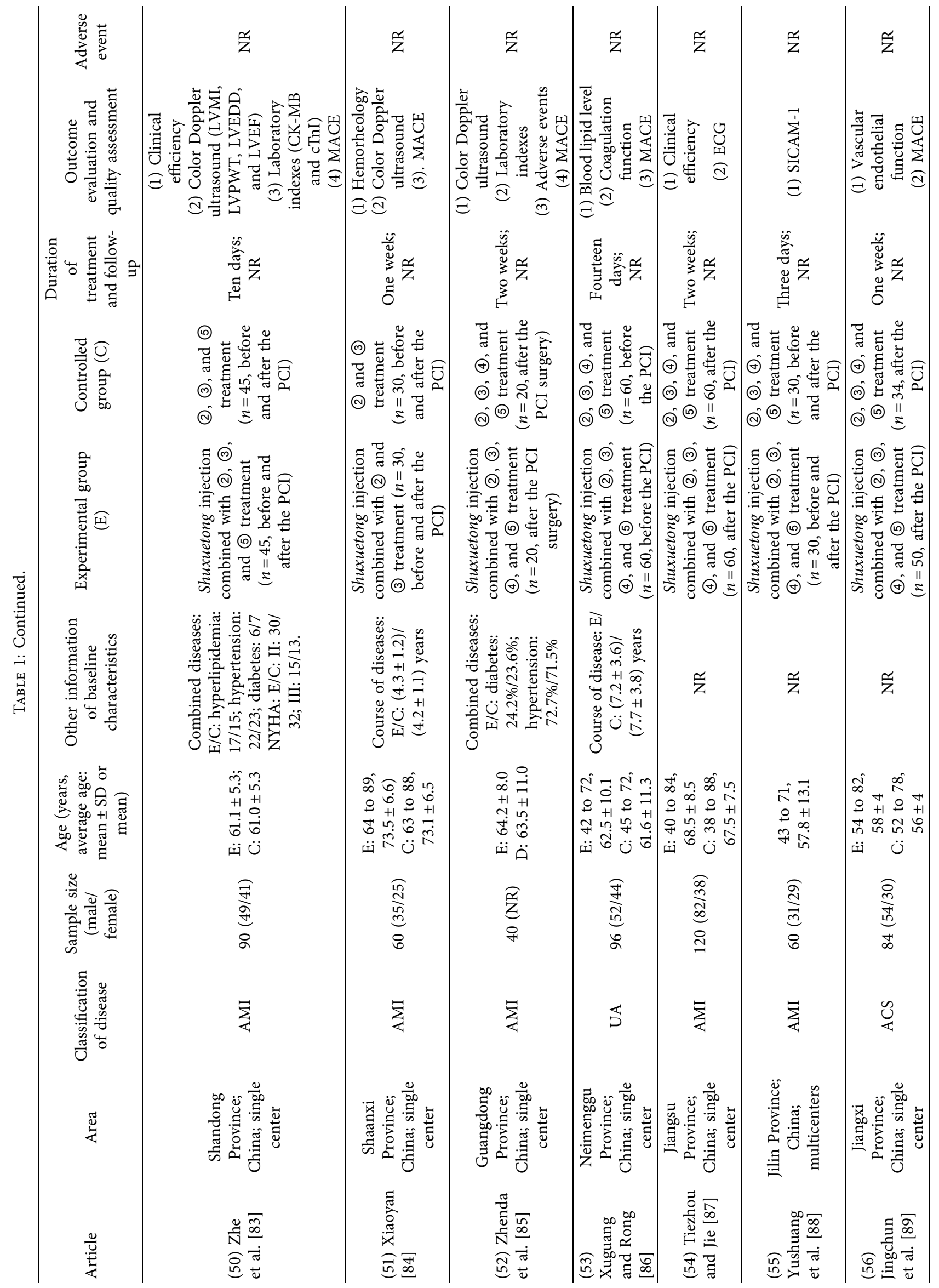




\begin{tabular}{|c|c|c|c|c|c|}
\hline 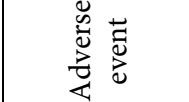 & 孚 & $\stackrel{\dot{\vec{\lambda}}}{\ddot{z}}$ & 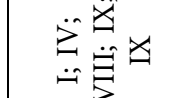 & 㐒 & 占 \\
\hline 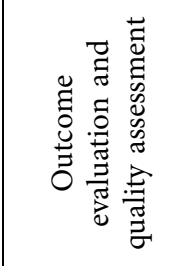 & 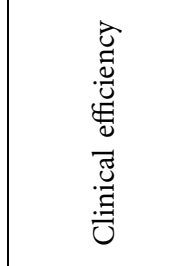 & 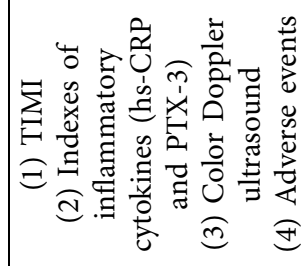 & 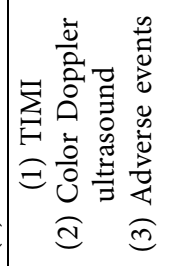 & 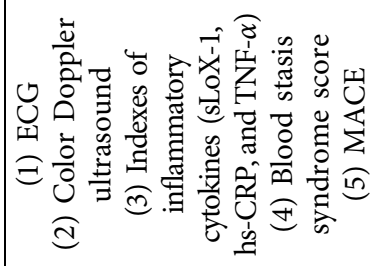 & 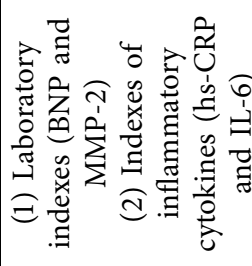 \\
\hline 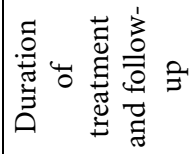 & 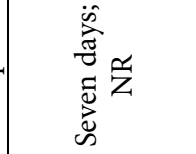 & 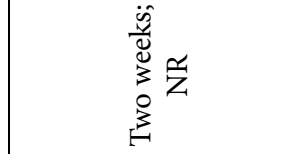 & 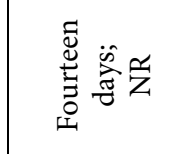 & 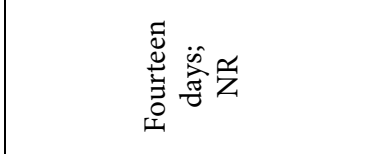 & 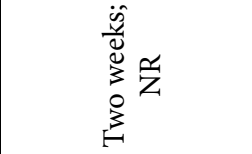 \\
\hline 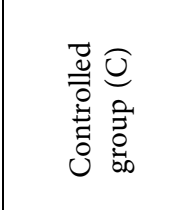 & 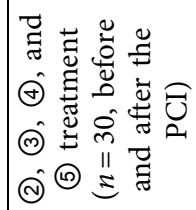 & 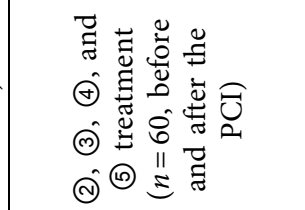 & 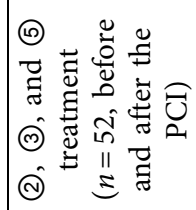 & 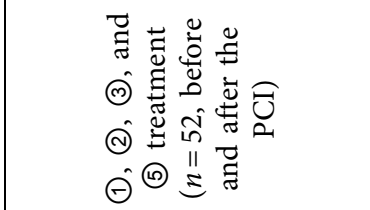 & 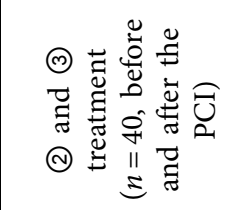 \\
\hline 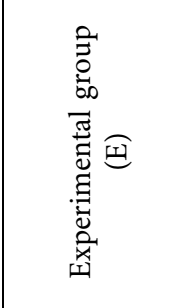 & 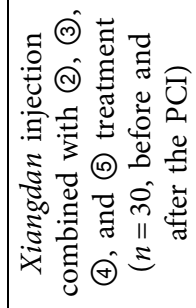 & 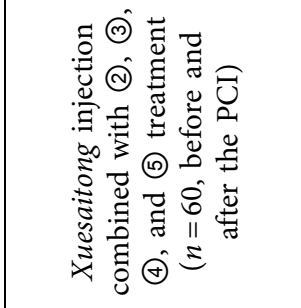 & 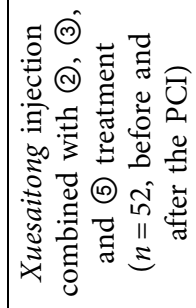 & 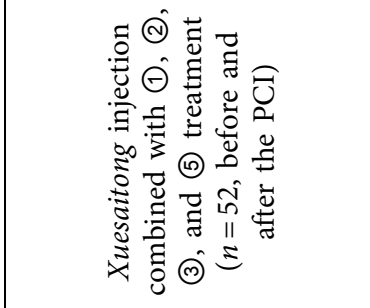 & 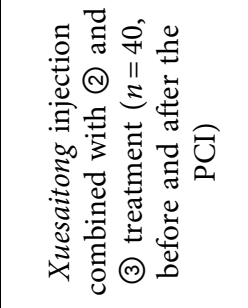 \\
\hline 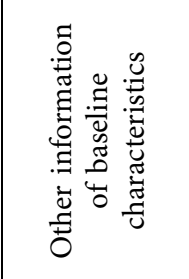 & 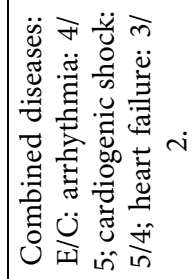 & 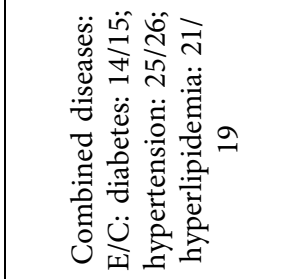 & 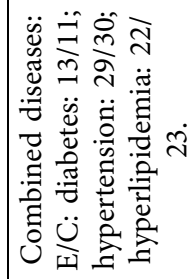 & 点 & 号 \\
\hline 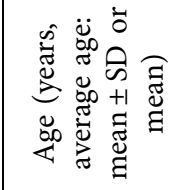 & 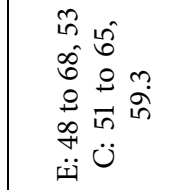 & 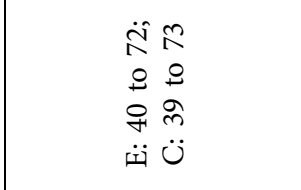 & 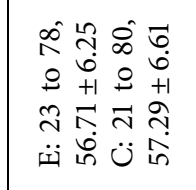 & 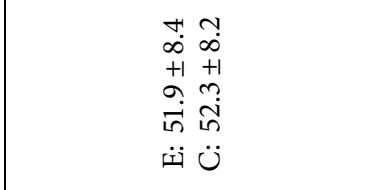 & 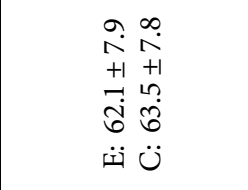 \\
\hline 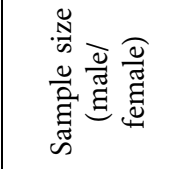 & 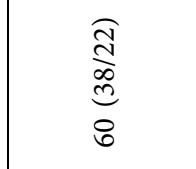 & 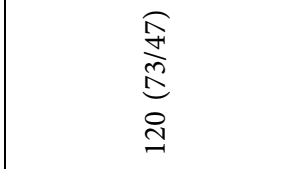 & 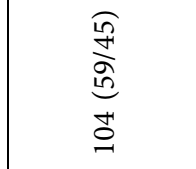 & 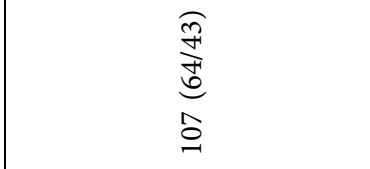 & 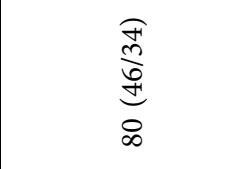 \\
\hline 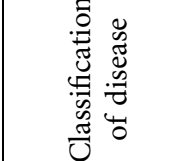 & $\sum$ & 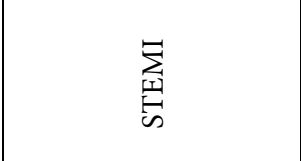 & 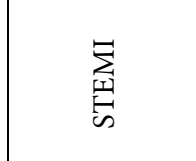 & $\sum_{\&}^{\xi}$ & $\sum$ \\
\hline 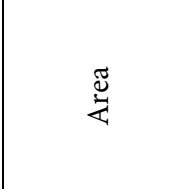 & 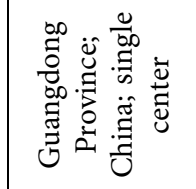 & 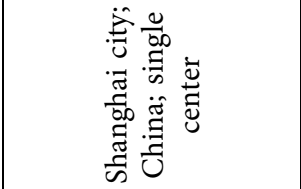 & 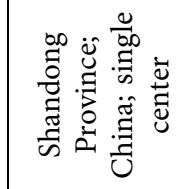 & 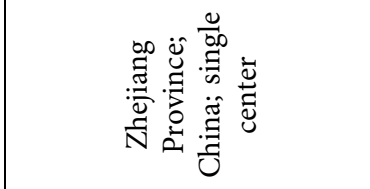 & 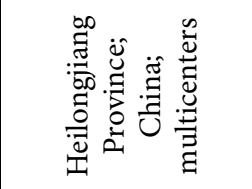 \\
\hline 节 & 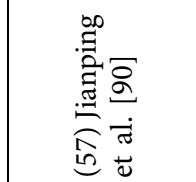 & 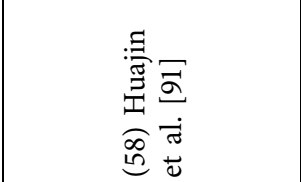 & 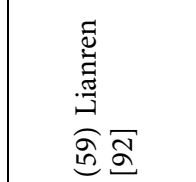 & 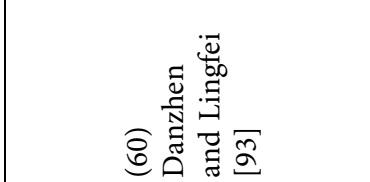 & 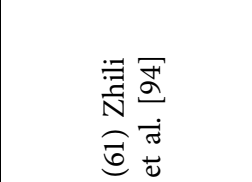 \\
\hline
\end{tabular}




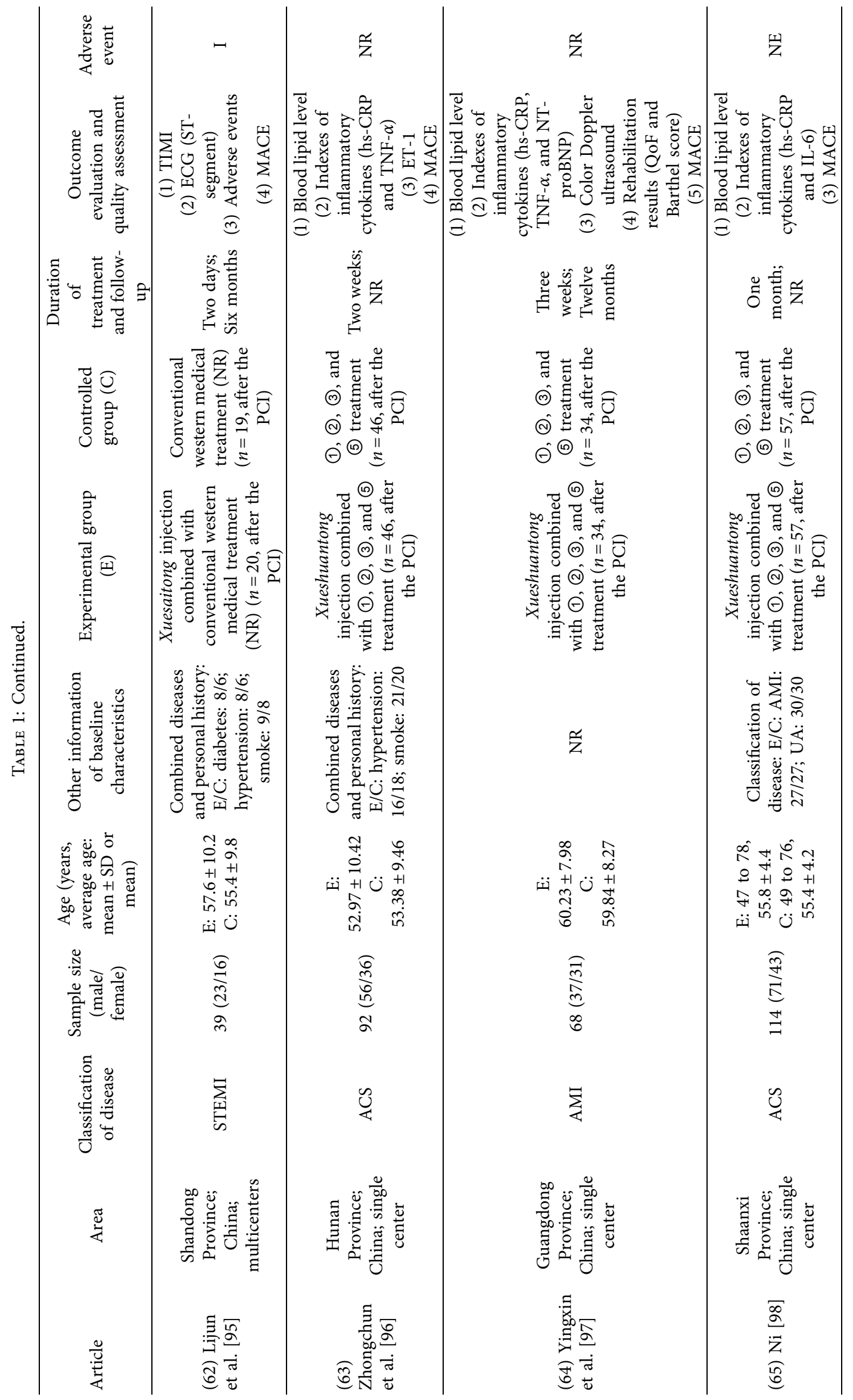




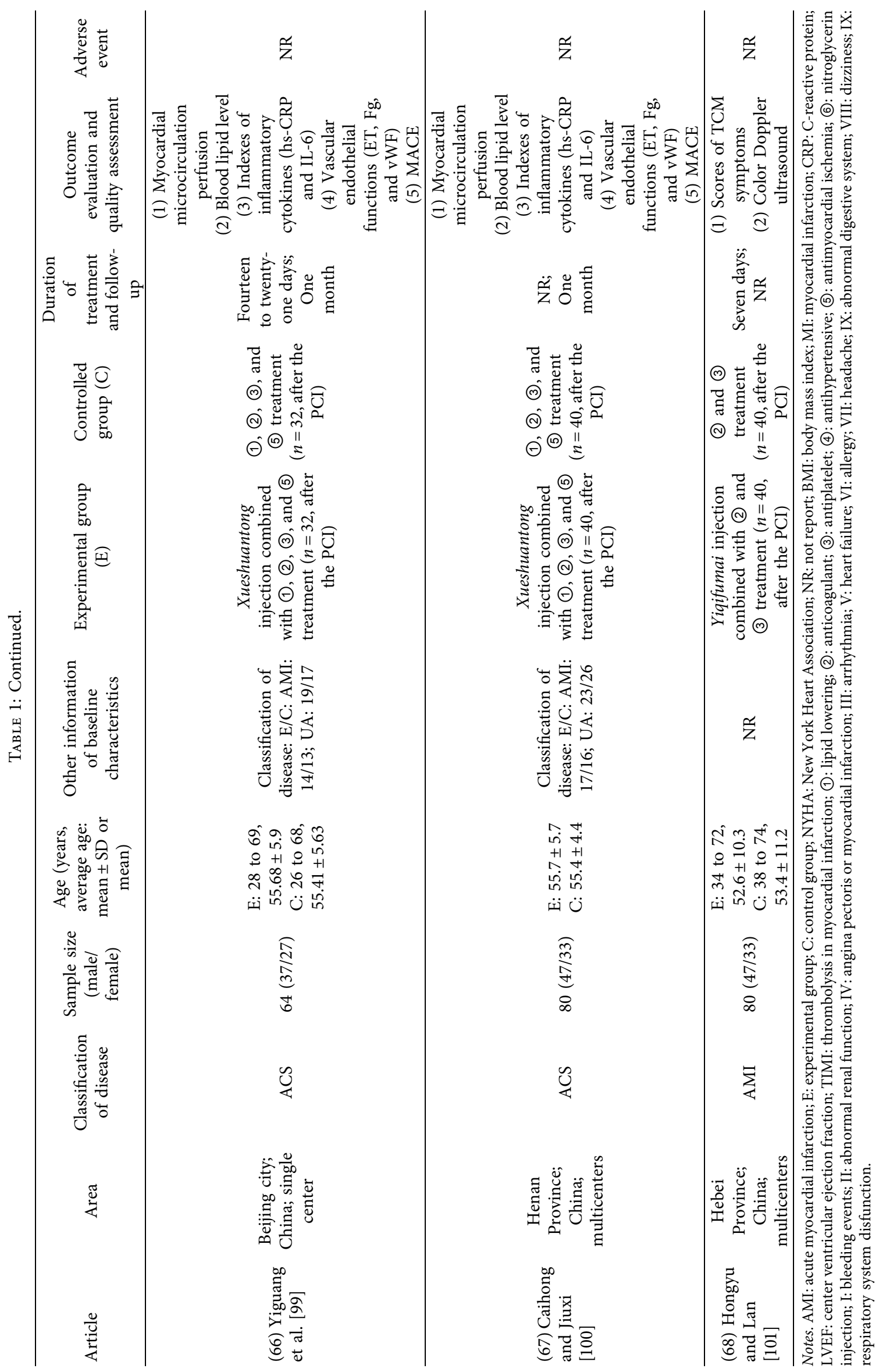




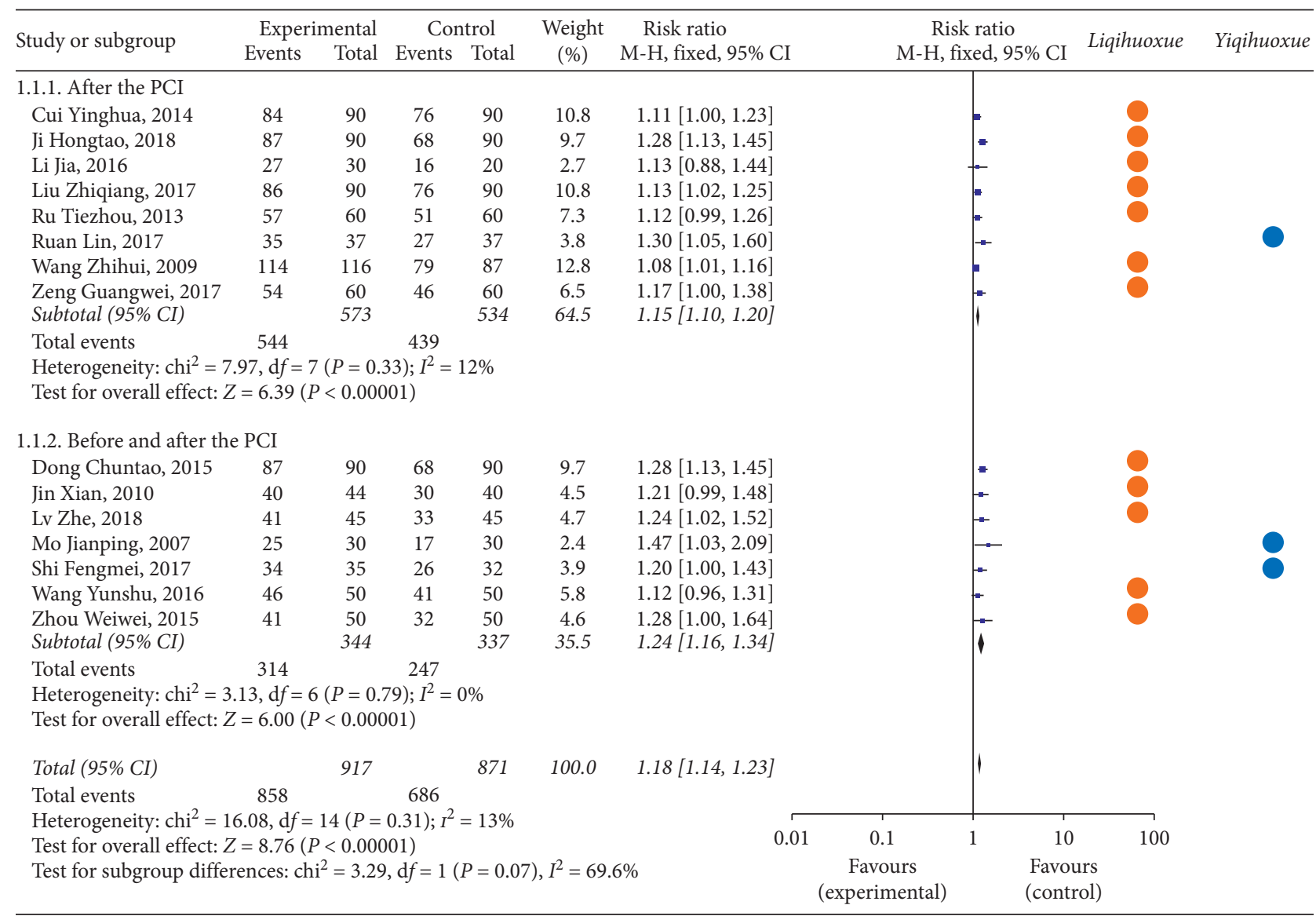

Figure 2: Forest plot of clinical efficiency of TCMI based on the time point of intervention and the effect of Liqihuoxue or Yiqihuoxue. Note. represents the TCMI with the effect of Liqihuoxue; represents the TCMI with the effect of Yiqihuoxue.

\begin{tabular}{|c|c|c|c|c|c|c|c|c|c|c|c|}
\hline \multirow{2}{*}{ Study or subgroup } & \multicolumn{2}{|c|}{ Experimental } & \multicolumn{2}{|c|}{ Control } & \multirow{2}{*}{$\begin{array}{c}\text { Weight } \\
(\%)\end{array}$} & \multirow{2}{*}{$\begin{array}{c}\text { Risk ratio } \\
\text { M-H, fixed, 95\% CI }\end{array}$} & \multirow{2}{*}{\multicolumn{3}{|c|}{$\begin{array}{c}\text { Risk ratio } \\
\text { M-H, fixed, } 95 \% \text { CI }\end{array}$}} & \multirow{2}{*}{ Liqihuoxue } & \multirow{2}{*}{ Yiqihuoxue } \\
\hline & Events & Total & Events & Total & & & & & & & \\
\hline \multicolumn{12}{|c|}{ 2.1.1. Before and after the PCI } \\
\hline Ding Faming, 2007 & 4 & 45 & 3 & 45 & 19.4 & $1.33[0.32,5.62]$ & & & - & & 0 \\
\hline Lv Zhe, 2018 & 0 & 45 & 1 & 45 & 9.7 & $0.33[0.01,7.97]$ & & & & 0 & \\
\hline Ma Xiaoyan, 2018 & 0 & 30 & 2 & 30 & 16.2 & $0.20[0.01,4.00]$ & & & & 0 & \\
\hline Subtotal (95\% CI) & & 120 & & 120 & 45.3 & $0.71[0.23,2.18]$ & & & & & \\
\hline Total events & \multirow{2}{*}{\multicolumn{10}{|c|}{$\begin{array}{l}\text { Total events } \\
\text { Heterogeneity: } \text { chi }^{2}=1.64, \mathrm{~d} f=2(P=0.44) ; I^{2}=0 \%\end{array}$}} & \\
\hline \multicolumn{9}{|c|}{ Test for overall effect: $Z=0.59(P=0.55)$} & & & \\
\hline \multicolumn{12}{|l|}{ 2.1.2. After the PCI } \\
\hline Li Hong, 2009 & 3 & 42 & 4 & 44 & 25.3 & $0.79[0.19,3.30]$ & & & & & 0 \\
\hline Zhao Dingxue, 2015 & 0 & 44 & 1 & 44 & 9.7 & $0.33[0.01,7.97]$ & & & & 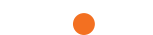 & \\
\hline Zhong Xinmin, 2015 & 2 & 36 & 3 & 35 & 19.7 & $0.65[0.12,3.65]$ & & & & 0 & \\
\hline Subtotal $(95 \%$ CI) & & 122 & & 123 & 54.7 & $0.66[0.23,1.85]$ & & & & & \\
\hline Total events & 5 & & 8 & & & & & & & & \\
\hline \multirow{2}{*}{\multicolumn{12}{|c|}{$\begin{array}{l}\text { Heterogeneity: } \mathrm{chi}^{2}=0.24, \mathrm{~d} f=2(P=0.89) ; I^{2}=0 \% \\
\text { Test for overall effect: } Z=0.80(P=0.42)\end{array}$}} \\
\hline & & & & & & & & & & & \\
\hline Total $(95 \%$ CI) & & 242 & & 243 & 100.0 & $0.68[0.32,1.46]$ & & & & & \\
\hline Total events & 9 & & 14 & & & & & & & & \\
\hline \multicolumn{12}{|c|}{$\begin{array}{l}\text { Total events } \\
\text { Heterogeneity: } \mathrm{chi}^{2}=1.91, \mathrm{~d} f=5(P=0.86) ; I^{2}=0 \%\end{array}$} \\
\hline \multirow{2}{*}{\multicolumn{7}{|c|}{$\begin{array}{l}\text { Test for overall effect: } Z=0.99(P=0.32) \\
\text { Test for subgroup differences: } \text { chi }^{2}=0.01, \mathrm{~d} f=1(P=0.91), I^{2}=0 \%\end{array}$}} & 0.01 & 0.1 & 1 & 100 & \\
\hline & & & & & & & & Favours & & ours & \\
\hline
\end{tabular}

FIgURE 3: Forest plot of all-cause mortality based on the time point of intervention and the effect of Liqihuoxue or Yiqihuoxue. 


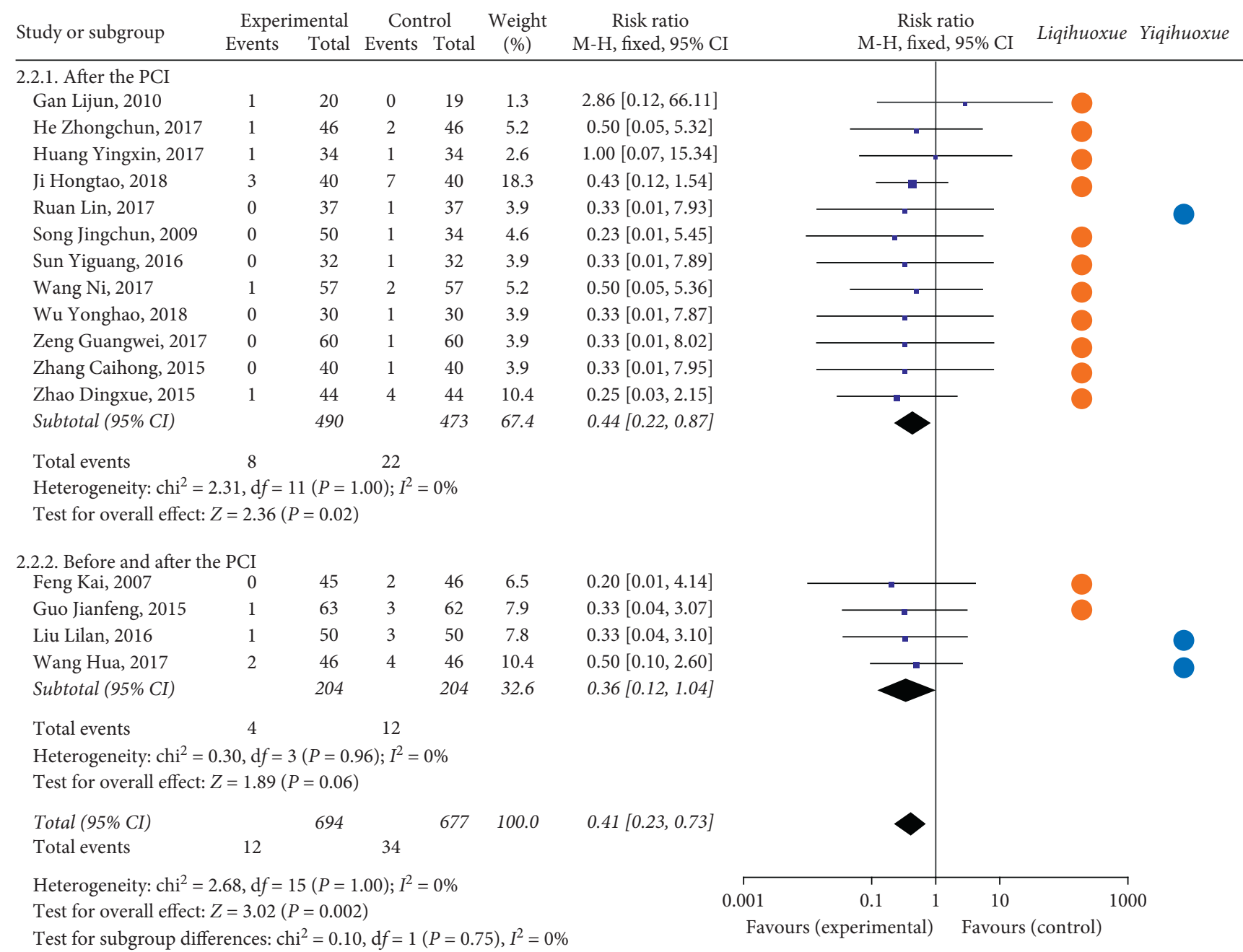

FIgURE 4: Forest plot of myocardial infarction based on the time point of intervention and the effect of Liqihuoxue or Yiqihuoxue.

$\left.P=0.86, I^{2}=0 \%\right)$, and the fixed-effects model was performed by the $M-H$ test.

(2) Myocardial Infraction. As for the myocardial infraction, twelve articles [34, 41, 50, 51, 76, 89, 95-100] with 993 participants received the treatment after the PCI compared with the 4 articles $[43,44,58,67]$ with 424 patients before and after the PCI (Figure 4). The result illustrated that the occurrence of myocardial infraction of the experimental group was lower than the controlled group based on the intervention of time point after the PCI $(\mathrm{RR}=0.44,95 \%$ $\mathrm{CI}=0.22$ to $0.87, P=0.02)$. The TCMI with the effect of Liqihuoxue [34, 50, 51, 58, 67, 76, 89, 95-100] showed the superiority on the time point after the PCI. The heterogeneity was also not found (after the PCI: $P=1.00, I^{2}=0 \%$; before and after the PCI: $P=0.96, I^{2}=0 \%$; overall: $P=1.00$, $I^{2}=0 \%$, and the fixed-effects model was performed by the $M-H$ test.

(3) Stenocardia. Twelve studies [34, 41, 46, 50, 51, 57, 89, 95, 96, 98-100] with 1,011 patients were treated after the PCI compared with the rest of four studies [39, 58, 67, 83] with 434 patients being treated before and after the PCI (Figure
5). The result showed that the occurrence of stenocardia for the experimental group was lower than the controlled group both on the two time points of intervention (after the PCI: $\mathrm{RR}=0.49,95 \% \mathrm{CI}=0.33$ to $0.72, P=0.0003$; before and after the PCI: $\mathrm{RR}=0.40,95 \% \mathrm{CI}=0.18$ to $0.89, P=0.02$; overall: $\mathrm{RR}=0.47,95 \% \mathrm{CI}=0.33$ to $0.66, P<0.0001)$. The TCMI with the effect of Liqihuoxue [34, 39, 50, 51, 57, 58, 67, 89, 95, 96, 98-100] showed the superiority on the time points before and after the PCI and after the PCI. No heterogeneity was found (after the PCI: $P=0.94, I^{2}=0 \%$; before and after the PCI: $P=0.61, I^{2}=0 \%$; overall: $P=0.97, I^{2}=0 \%$ ), and the fixed-effects model was performed by the $M-H$ test.

(4) Arrhythmia. Figure 6 illustrated the outcome of arrhythmia. Three studies [41, 46, 71] with 216 patients received the treatment after the PCI compared with the five studies [39, 42-44, 93] with 567 patients received the treatment before and after the PCI. The result showed that the occurrence of arrhythmia for the experimental group was lower than the controlled group on the time points before and after the PCI $(\mathrm{RR}=0.33,95 \% \mathrm{CI}=0.2$ to 0.56 , $P<0.001)$. Both TCMI with the effect of Liqihuoxue [39, 42, 93] and Yiqihuoxue [41, 43, 44, 46, 71] showed the 


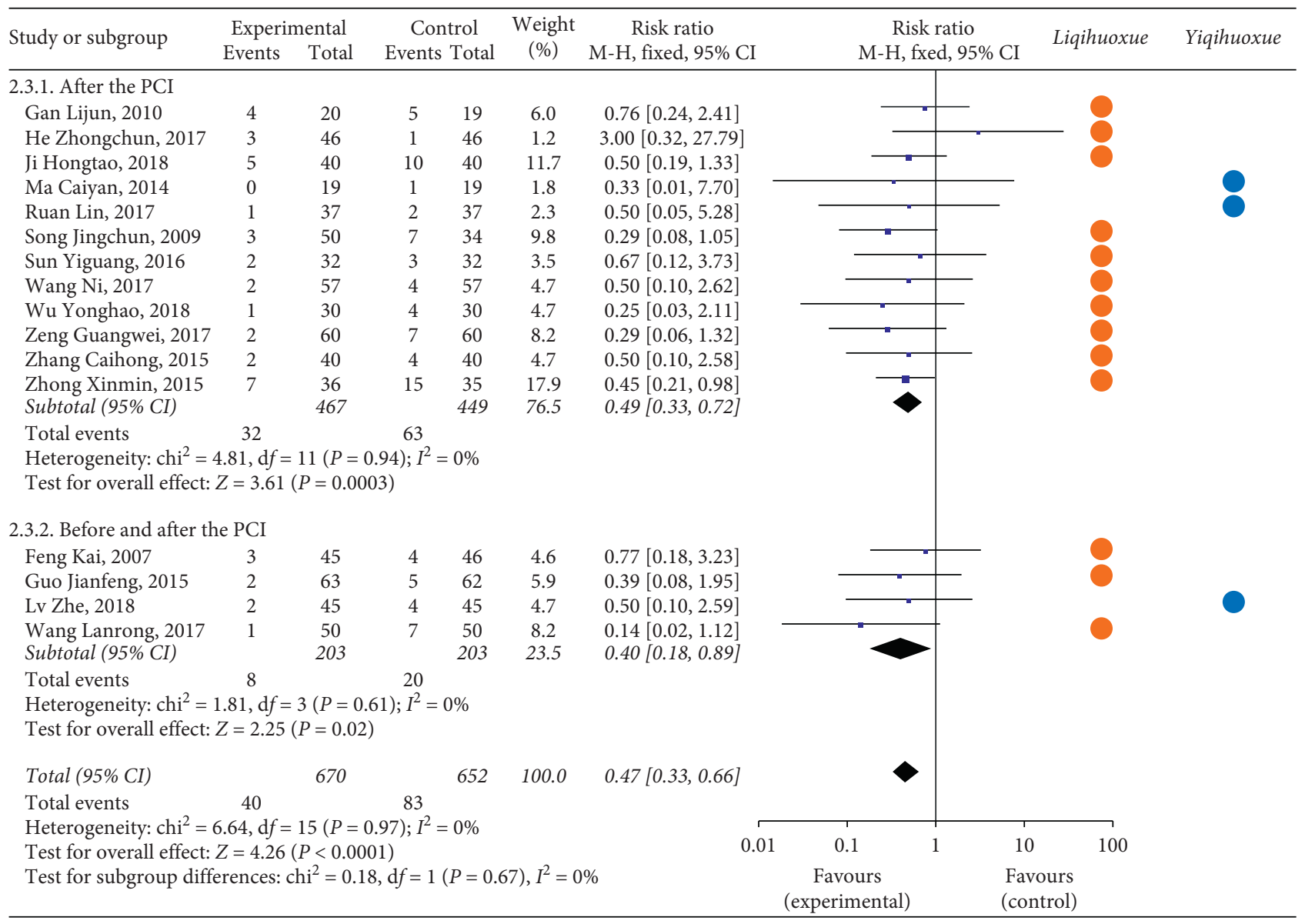

Figure 5: Forest plot of stenocardia based on the time point of intervention and the effect of Liqihuoxue or Yiqihuoxue.

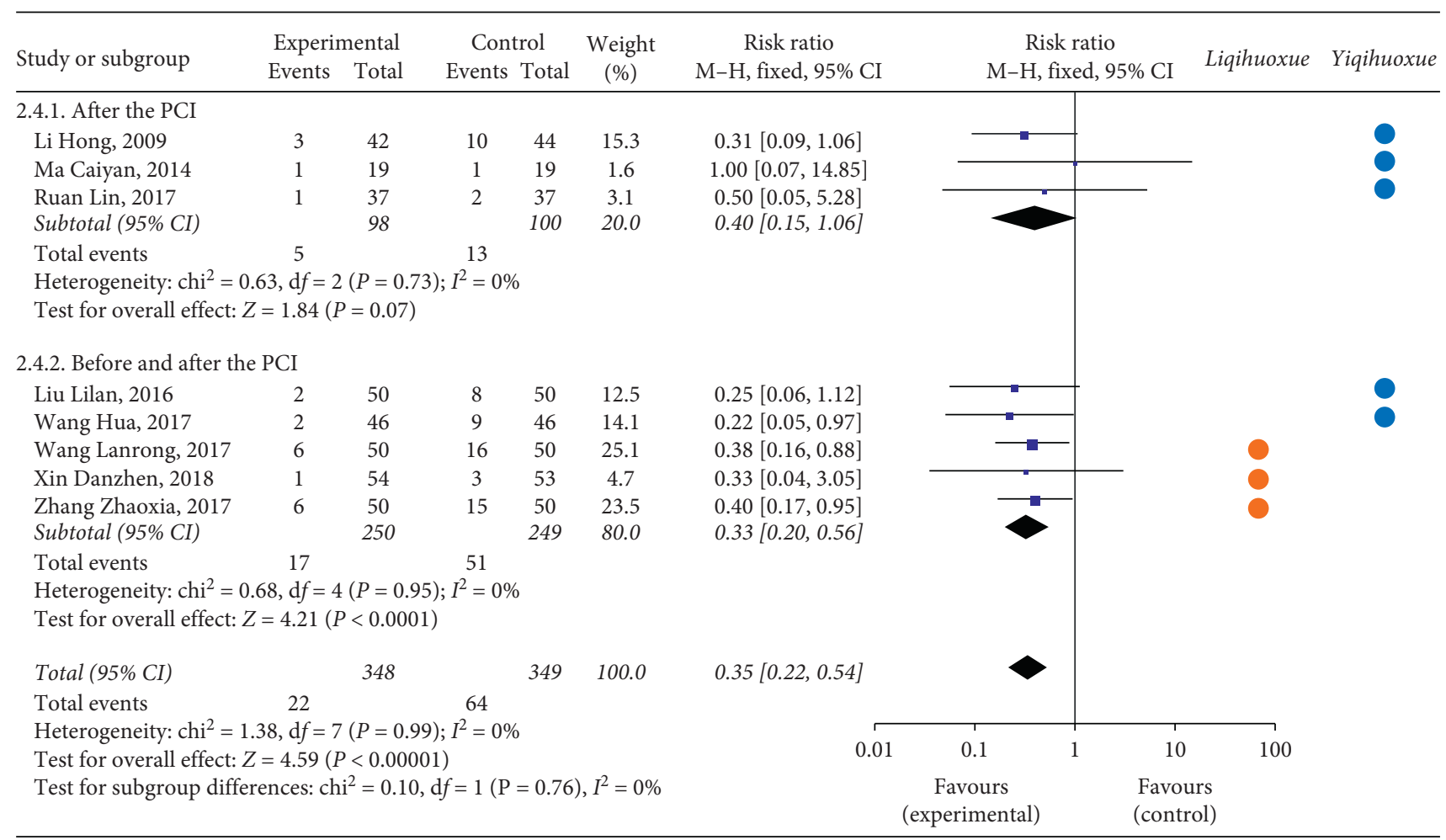

FIgURE 6: Forest plot of arrhythmia based on the time point of intervention and the effect of Liqihuoxue or Yiqihuoxue. 


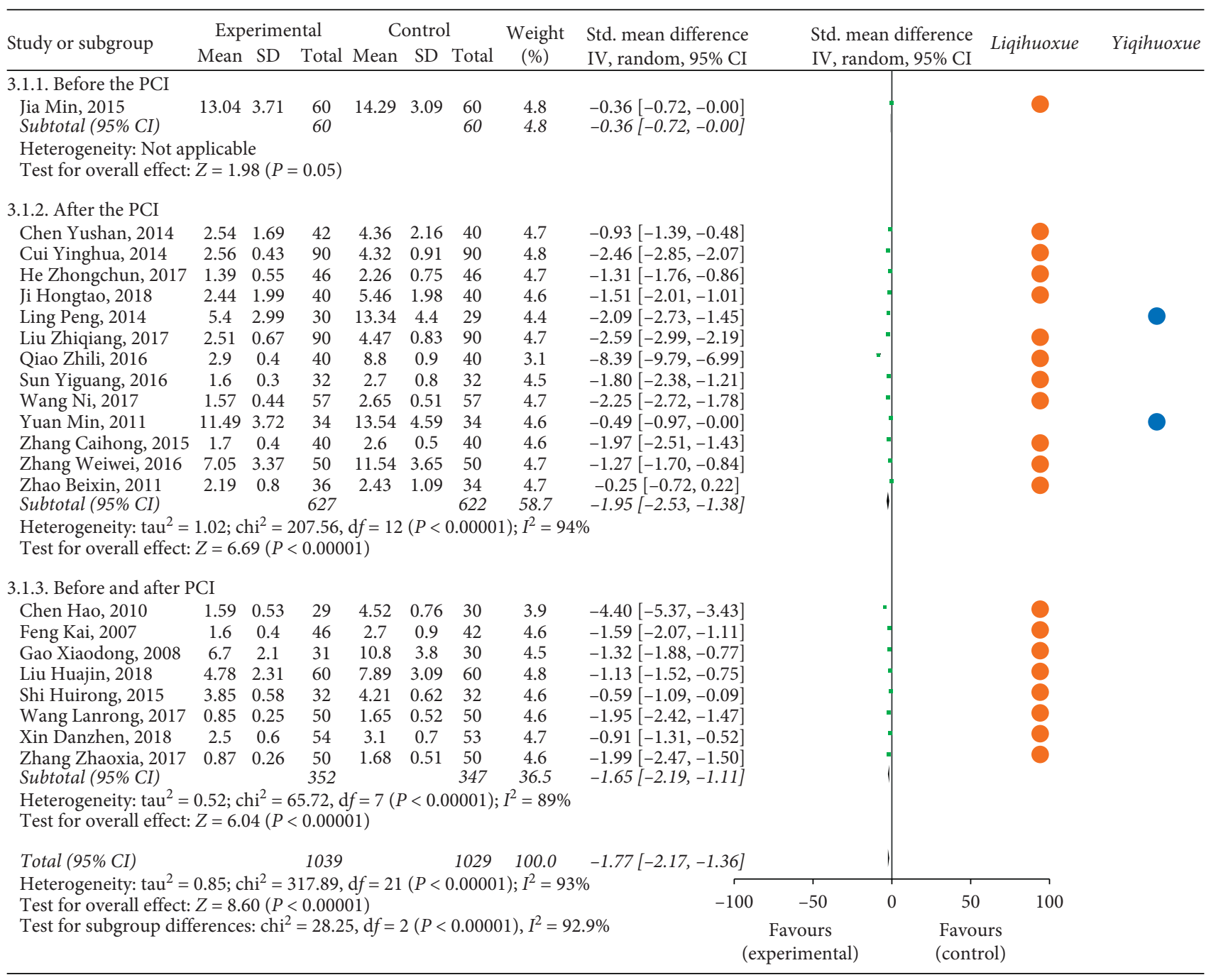

FIGURE 7: Forest plot of hs-CRP based on the time point of intervention and the effect of Liqihuoxue or Yiqihuoxue.

superiority on the intervention of time points before and after the PCI. No heterogeneity was found (after the PCI: $P=0.73, I^{2}=0 \%$; before and after the PCI: $P=0.95, I^{2}=0 \%$; overall: $\left.P=0.99, I^{2}=0 \%\right)$, and the fixed-effects model was performed by the $M-H$ test.

In a word, even though the TCMI combined with western medicine showed the advantage on some indicators of the MACE compared with western medicine alone, the result still could not recommend the best applying point of TCMI during the perioperative period of PCI for patients with ACS.

3.4.3. Inflammatory Factors. Figures 7 and 8 illustrate the inflammatory factors (hs-CRP and IL-6) of patients with ACS after the treatment of experimental group and controlled group based on the effect of Yiqihuoxue or Liqihuoxue and the time points of intervention.

(1) hs-CRP. A total of 13 studies [34, 37, 45, 47, 52, 53, 59, $62,94,96,98-100]$ with 1,249 patients were treated after the PCI compared with 8 studies [36, 39, 42, 63, 66, 67, 91, 93] with 699 patients being treated before and after the PCI (Figure 7). The result of meta-analysis indicated that the level of hs-CRP for the experimental group was lower than the controlled group (after the PCI: Std. MD $=-1.95,95 \%$ $\mathrm{CI}=-2.53$ to $-1.38, P<0.001$; before and after the PCI: Std. $\mathrm{MD}=-1.65,95 \% \mathrm{CI}=-2.19$ to $-1.11, P<0.001$; overall: Std. $\mathrm{MD}=-1.77,95 \% \mathrm{CI}=-2.17$ to $-1.36, P<0.001)$. The TCMI with the effect of Liqihuoxue [34, 36, 37, 39, 42, 52, $53,59,62,63,66,67,91,93,94,96,98-100]$ was superior to the Yiqihuoxue $[45,47]$ during the perioperative period of PCI. But it still could not recommend the best time point of intervention during the perioperative period of PCI. Significant statistical heterogeneity was found (after the PCI: $P<0.01, I^{2}=99 \%$; before and after the PCI: $P<0.01$, $I^{2}=97 \%$; overall: $\left.P<0.01, I^{2}=98 \%\right)$, and the random-effects model was performed by the $I V$ test. The subgroup analysis was applied to explore the source of heterogeneity based on the classification of area (north or south of China), level of hospitals (three A hospital or not), and sample size of studies (more than 100 or less than 100). The result indicated that the level of hospitals might was the 


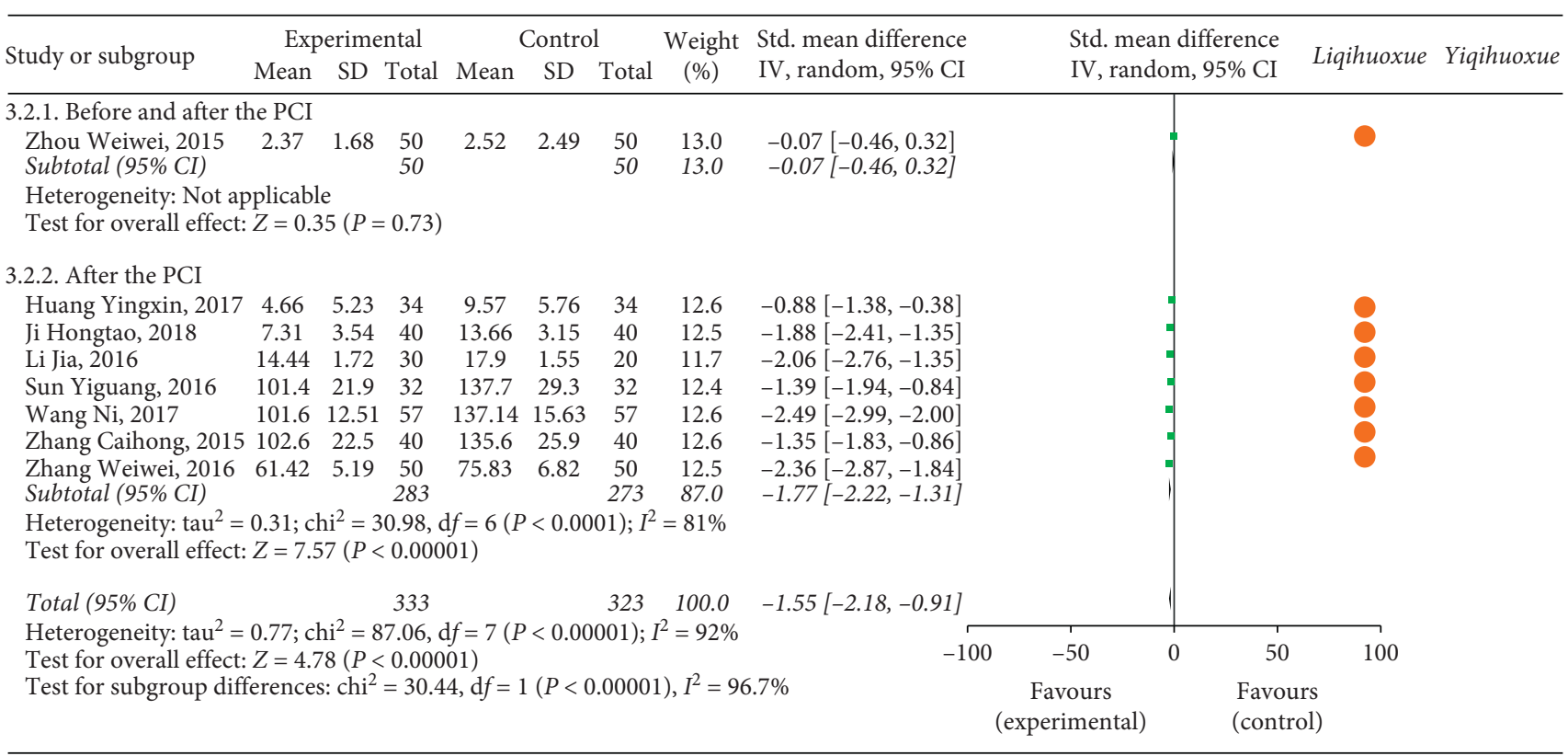

FIgURE 8: Forest plot of IL-6 based on the time point of intervention and the effect of Liqihuoxue or Yiqihuoxue.

source of heterogeneity (see Figures S3-S5 in the Supplementary Materials).

(2) IL-6. Seven articles [34, 35, 53, 97-100] with 556 patients received the treatment after the PCI compared with only 1 article [73] with 100 patients received the treatment before and after the PCI (Figure 8). The result showed that the IL- 6 for the experimental group was lower than the controlled group on the time point after the PCI (Std. $\mathrm{MD}=-1.77,95 \% \mathrm{CI}=-2.22$ to $-1.31, P<0.001$ ), and the Liqihuoxue [34, 35, 53, 73, 97-100] was the most frequent effect of TCMI in this part. Obvious heterogeneity was found (after the PCI: $P<0.01, I^{2}=81 \%$; overall: $P<0.01$, $I^{2}=92 \%$ ), and the random-effects model was performed by the $I V$ test. The subgroup analysis was also conducted to explore the source of heterogeneity based on the classification of area (north or south of China), level of hospitals (three A hospital or not), and sample size of studies (more than 100 or less than 100). But the result could not reveal the source of heterogeneity (see Figures S6-S8 in the Supplementary Materials).

3.5. Adverse Events. From the included researches, the report of potential adverse events mainly concentrated on bleeding events $[37,46,58,60,62,95]$, kidney disfunction $[41,51]$, angina pectoris or myocardial infarction [41-43, $91,92]$, arrhythmia $[41-43,46]$, respiratory system disfunction $[41,92]$, heart failure $[46,91]$, allergy $[51,57,62]$, headache [57], digestive system disfunction [92], and dizziness [91, 92]. Although there was no evidence that adverse events were directly caused by the application of TCMI, the bleeding events including gastrointestinal and gingival bleeding, haemoptysis, puncture point hematoma, and subcutaneous congestion were the most relevant events.
3.6. Publication Bias. We applied the RR or $\mathrm{MD}$ as the midpoint to draw the funnel plot (Figure 9). The publication bias was evaluated in the funnel plot by comparing the symmetry of included studies on clinical efficiency, MI, stenocardia, and hs-CRP. Each outcome indicator should include more than 10 studies. The funnel plot was symmetrical in visual for clinical efficiency, MI, and stenocardia, while not for hs-CRP. The statistical method of Egger's and Begg's test was conducted and further verified the publication bias by the software Stata. The results of Egger's and $B e g g$ 's test indicated that the publication bias did not exist in clinical efficiency (Egger's test $(t=0.05, P=0.962>0.05)$; Begg's test $(z=0.25, P=0.805>0.05))$ and hs-CRP (Egger's test $(t=-0.89, \quad P=0.389>0.05) ; \quad B e g g$ 's test $(z=1.86$, $P=0.063>0.05))$. However, the MI (Egger's test $(t=-5.73$, $P=0.001) ; B e g g$ 's test $(z=2.60, P=0.009))$ and stenocardia (Egger's test $(t=-4.08, P=0.001) ; B e g g$ 's test $(z=2.28$, $P=0.023)$ ) obtained the publication bias (see Figures S9-S12 in the Supplementary Materials).

\section{Discussion}

As one of the diseases that endanger human health and life seriously, ACS has aroused extensive attention all over the world [5]. The PCI has been widely applied in the treatment of ACS, and the prognosis has dramatically improved [18]. However, some PCI-related problems, such as no-reflow, ischemia-reperfusion injury, PMI, in-stent restenosis, and stent thrombosis, are difficult to avoid. Previous research studies illustrated that TCMI had a good effect on preventing arrhythmia and reperfusion injury, improving heart function, and protecting myocardium [22]. However, there was insufficient medical evidence for the TCMI in patients with ACS based on the effective classification of Liqihuoxue and Yiqihuoxue. This study was based on the PRISMA statement, focusing on the efficacy and safety of TCMI for ACS with the 


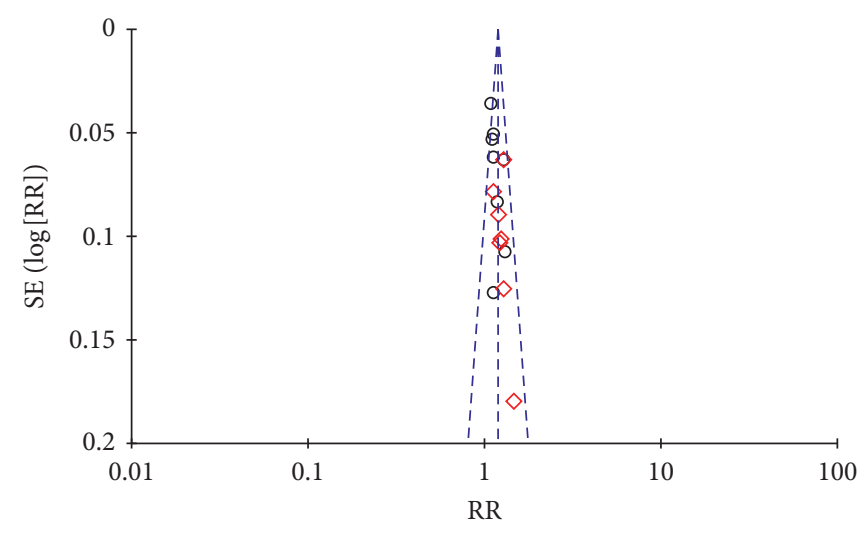

Subgroups

○ Intervention after the PCI surgery

$\diamond$ Intervention before and after the PCI surgery

(a)

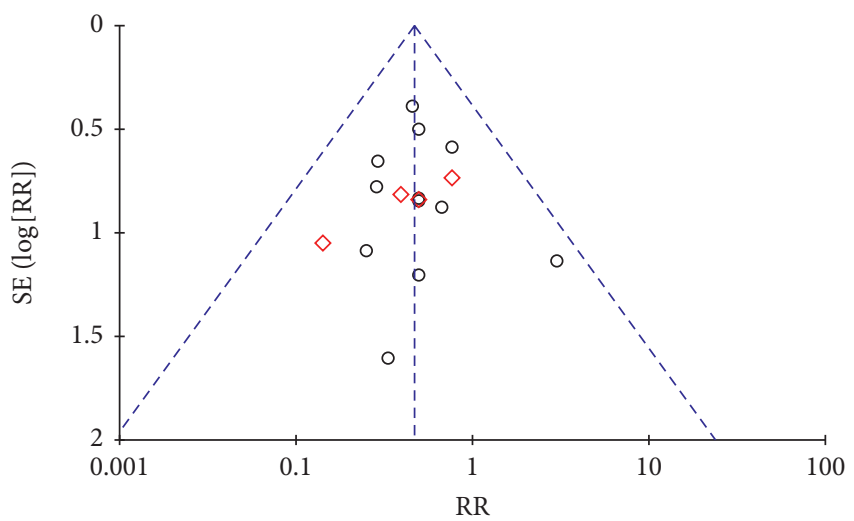

Subgroups

O After the surgery

$\diamond$ Before and after the surgery

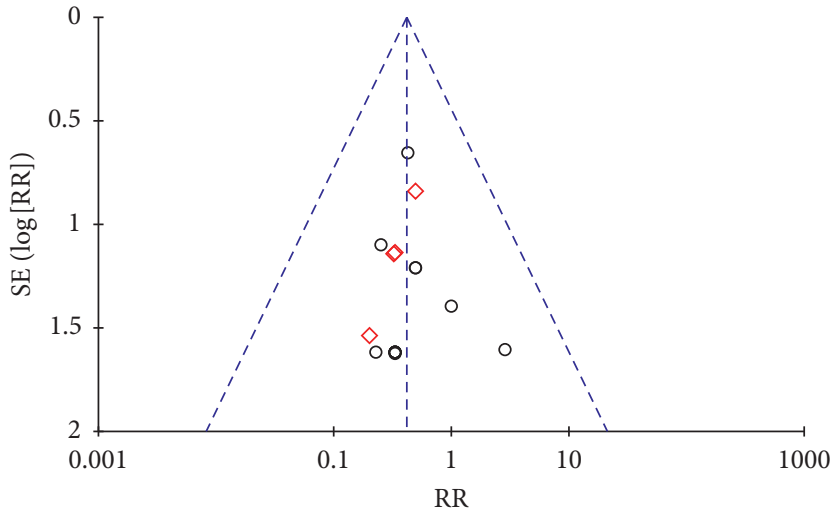

Subgroups

$\bigcirc$ After the surgery

$\diamond$ Before and after the surgery

(b)

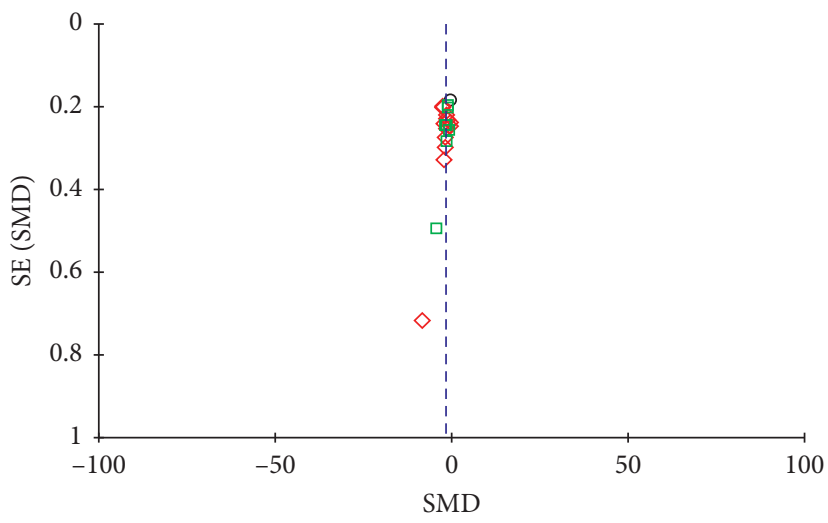

Subgroups

○ Before the surgery

$\diamond$ After the surgery

$\square$ Before and after surgery

(d)

(c)

Figure 9: The funnel plot of (a) clinical efficiency, (b) MI, (c) stenocardia, and (d) hs-CRP.

effect of Yiqihuoxue or Liqihuoxue and the time points of intervention during the perioperative period of PCI. The characteristics of TCMI and the precision of intervention are well illustrated.

A total of 68 articles with 6,043 patients were enrolled in this meta-analysis. The result of meta-analysis showed that the clinical efficiency of TCMI combined with western medicine (experimental group) was superior to the western medicine alone (controlled group) on patients with ACS during the perioperative period of PCI (before the PCI, before and after the PCI, or both), and the TCMI with the effect of Liqihuoxue was the relatively better choice. The result of MACE illustrated that the occurrence of MI, stenocardia, and arrhythmia for the experimental group was lower than the controlled group (MI and stenocardia: time points before the PCI, before and after the PCI, or both; arrhythmia: time points before and after PCI). However, the occurrence of all-cause mortality did not prove the advantage of TCMI. The TCMI with the effect of Liqihuoxue was the relatively better choice for the prevention of MACE based on the evaluation of classification. The result of meta-analysis for inflammatory factors showed that the level of hs-CRP and IL- 6 for the experimental group was lower than the controlled group (hs-CRP: in the period of before the PCI, before and after the PCI, or both; IL-6: after the PCI) and both TCMI with the effect of Liqihuoxue and Yiqihuoxue has shown the superiority. The heterogeneity of some indicators (hs-CRP and IL-6) was extremely obvious, and the result of subgroup analysis indicated the level of hospitals might be the source of heterogeneity for hs-CRP. After each included study was excluded individually based on the procedure of sensitivity analysis, the majority of the combined effects were relatively close and stable.

The publication bias existed in this research after Egger's and Begg's tests. It might come from the following reasons: (a) some authors tended to deliver positive results to editors while prejudiced negative results [102]; (b) some editors or reviewers had a preference to positive results while cavilled to negative results to some extent [103]; (c) government 
funding researches had more possibilities to be published in some magazines than receiving private or company funding [104]. The meta-analysis would overstate the degree of association between treating effects and risk factors because of the publication bias, bringing mistakes for clinical therapy or health decision-making.

Numerous previous systematic reviews and meta-analyses have been published to confirm the clinical efficacy and safety of TCM for the treatment of CHD. However, there still remained some problems. Firstly, some of them only focused on the broad category of CHD without evaluating the specific type of disease, leading to the restriction of clinical application $[105,106]$. Secondly, some of them did not classify the category and dosage of TCM, leading to more confounding factors and high risk of bias [107]. Thirdly, some studies did not highlight the precise time point of intervention for TCMI during the perioperative period of PCI [108, 109]. Compared with previous research studies, the characteristics of our research were clearly classification of TCMI (the effect of Yiqihuoxue and Liqihuoxue), accurate selection of disease types from the $\mathrm{CHD}$, and precise time point of intervention during the perioperative period of PCI (before the PCI, before and after the PCI, after the PCI, and overall).

It should be noted that some limitations did exist as follows. Firstly, all included studies were conducted in different hospitals in China, which might bring the regional and cultural bias based on the different clinical abilities of ACS diagnosis and PCI treatment. Secondly, the included RCTs had flaws caused by human baseline risk factors (all patients were Chinese), incomplete methodological design of trials (lack of blinding method), and small sample size (less than 30 patients per group). Thirdly, some results showed significant heterogeneity, which might be due to the sample size, the different experimental regions in China, medicine application and dose, publication years, and the duration of treatment. The lower quality of included RCTs restricted the promotion of evidence. Fourthly, the random-effects model was established to pool data, which might not provide the exact and stable conclusion based on this situation.

The report of adverse events of TCM, including the TCMI, has always been a hotspot issue in clinical practice. Recently published retrospective research, which reviewed the data from 10,000 heart failure patients, found that Salvia miltiorrhiza/Danshen might increase the risk of bleeding and death [110]. Some articles emphasized that the occurrence of adverse events was actually related to the nonstandardized use of Chinese medicine in western medical hospitals so that the clinical value of TCM should not be negated completely. The precise treatment and safety evaluation of TCM are essential for the development of TCM, and this meta-analysis could provide evidence-based support and guidance.

\section{Conclusions}

Our research provides a beneficial and promising result for the application of TCMI (Liqihuoxue or Yiqihuoxue) combined with western medicine on patients with ACS during the perioperative period of PCI. This combined therapy can provide assistance for improving clinical efficiency, reducing the incidence rate of MACE, and lowering the level of inflammatory factors. We did not find the optimal time point of intervention during the perioperative period of PCI. Although the application of TCMI with the effect of Liqihuoxue obtained support from this research, the effect of Liqihuoxue or Yiqihuoxue for TCMI still needs more evidence from the standard, multicentre, double-blind RCTs in the future. The precise application of TCMI during the perioperative period of PCI will be one of the new directions for TCM in the future.

\section{Conflicts of Interest}

All authors declare that there are no conflicts of interest regarding the publication of this paper.

\section{Acknowledgments}

The authors would like to acknowledge Professor Yan Liu from Dongzhimen Hospital of Beijing University of Chinese Medicine, for his guidance and advice in analysis and improvement of data. This study was funded by grants from the National Key R\&D Program of China (2017YFC1700400 and 2017YFC1700402) and the National Science Fund for Distinguished Young Scholars (81725024).

\section{Supplementary Materials}

Figure S1: risk of bias graph. Figure S2: risk of bias summary. Figure S3: subgroup analysis of hs-CRP based on the classification of area. Figure S4: subgroup analysis of hs-CRP based on the classification of levels of hospital. Figure S5: subgroup analysis of hs-CRP based on the classification of sample size. Figure S6: subgroup analysis of IL-6 based on the classification of area. Figure S7: subgroup analysis of IL-6 based on the classification of levels of hospital. Figure S8: subgroup analysis of IL-6 based on the classification of sample size. Figure S9: Egger's and Begg's test for clinical efficiency. Figure S10: Egger's and Begg's test for hs-CRP. Figure S11: Egger's and Begg's test for MI. Figure S12: Egger's and Begg's test for stenocardia. Figure S13: specification of Danhong injection. Figure S14: specification of Safflower yellow injection. Figure S15: specification of Kudiezi injection. Figure S16: specification of Dazhuhongjingtian injection. Figure S17: specification of Shuxuetong injection. Figure S18: specification of Xuesaitong injection. Figure S19: specification of Guanxinning injection. Figure S20: specification of Shengmai injection. Figure S21: specification of Shenmai injection. Figure S22: specification of Xiangdan injection. Figure S23: specification of Gualoupi injection. Figure S24: specification of Xueshuantong injection. Figure S25: specification of Safflower injection. Figure S26: specification of Danshen injection. Figure S27: specification of Dengzhanhuasu injection. Figure S28: specification of Yiqifumai injection. Table S1: table of the risk of bias summary. Table S2: the detailed information of included TCMI. (Supplementary Materials) 


\section{References}

[1] T. Adam, "Acute coronary syndromes," BMJ, vol. 351, p. h5153, 2015.

[2] F. Crea, R. K. Binder, and T. F. Lüscher, "The year in cardiology 2017: acute coronary syndromes," European Heart Journal, vol. 39, no. 13, pp. 1054-1064, 2018.

[3] K. Smolina, F. L. Wright, M. Rayner, and M. J. Goldacre, "Determinants of the decline in mortality from acute myocardial infarction in England between 2002 and 2010: linked national database study," $B M J$, vol. 344 , no. 2, p. d8059, 2012.

[4] Emergency medicine branch of Chinese medical association, "Emergency rapid diagnosis and treatment of guidelines acute coronary syndrome," Chinese Journal of Emergency Medicine, vol. 36, no. 4, pp. 207-214, 2016.

[5] M. Liyuan, W. Yazhe, and W. Wen, "Interpretation of the report on cardiovascular diseases in China (2017)," Chinese Journal of Cardiovascular Medicine, vol. 23, no. 1, pp. 1-20, 2018.

[6] E. W. Carlton, M. Than, L. Cullen, A. Khattab, and K. Greaves, "“Chest pain typicality" in suspected acute coronary syndromes and the impact of clinical experience," The American Journal of Medicine, vol. 128, no. 10, pp. 1109-1116, 2015.

[7] K. Thygesen, J. S. Alpert, A. S. Jaffe et al., "Fourth universal definition of myocardial infarction (2018)," Circulation, vol. 138, no. 20, pp. e618-e651, 2018.

[8] X. S. Li, S. Obeid, R. Klingenberg et al., "Gut microbiotadependent trimethylamine $\mathrm{N}$-oxide in acute coronary syndromes: a prognostic marker for incident cardiovascular events beyond traditional risk factors," European Heart Journal, vol. 38, pp. 814-824, 2017.

[9] P. Jakob, T. Kacprowski, S. Briand-Schumacher et al., "Profiling and validation of circulating microRNAs for cardiovascular events in patients presenting with ST-segment elevation myocardial infarction," European Heart Journal, vol. 38, pp. 511-515, 2016.

[10] M. Margaritis, F. Sanna, G. Lazaros et al., "Predictive value of telomere length on outcome following acute myocardial infarction: evidence for contrasting effects of vascular vs. blood oxidative stress," European Heart Journal, vol. 38, no. 41, pp. 3094-3104, 2017.

[11] F. Costa, D. van Klaveren, S. James et al., "Derivation and validation of the predicting bleeding complications in patients undergoing stent implantation and subsequent dual antiplatelet therapy (PRECISE-DAPT) score: a pooled analysis of individual-patient datasets from clinical trials," The Lancet, vol. 389, no. 10073, pp. 1025-1034, 2017.

[12] E. Abu-Assi, S. Raposeiras-Roubin, P. Lear et al., "Comparing the predictive validity of three contemporary bleeding risk scores in acute coronary syndrome," European Heart Journal: Acute Cardiovascular Care, vol. 1, no. 3, pp. 222-231, 2012.

[13] P. Damman, A. W. van't Hof, J. M. Ten Berg et al., "2015 ESC guidelines for the management of acute coronary syndromes in patients presenting without persistent st-segment elevation: comments from the Dutch acs working group," Netherlands Heart Journal, vol. 25, no. 3, pp. 181-185, 2017.

[14] D. Sibbing, D. Aradi, and C. Jacobshagen, "Guided de-escalation of antiplatelet treatment in patients with acute coronary syndrome undergoing percutaneous coronary intervention (TROPICAL-ACS): a randomised, open-label, multicentre trial," The Lancet, vol. 390, pp. 1747-1757, 2017.
[15] J. Qiao, X. Zhang, J. Zhang et al., "Comparison between fondaparinux and low-molecular-weight heparin in patients with acute coronary syndrome: a meta-analysis," Cardiology, vol. 133, no. 3, pp. 163-172, 2015.

[16] R. Bugiardini, E. Cenko, B. Ricci et al., "Comparison of early versus delayed oral $\beta$ blockers in acute coronary syndromes and effect on outcomes," The American Journal of Cardiology, vol. 117, no. 5, pp. 760-767, 2016.

[17] S. Windecker, P. Kolh, F. Alfonso et al., "2014 ESC/EACTS guidelines on myocardial revascularization: the task force on myocardial revascularization of the European society of cardiology (ESC) and the European association for cardiothoracic surgery (EACTS) developed with the special contribution of the European association of percutaneous cardiovascular interventions (EAPCI)," European Heart Journal, vol. 35, no. 37, pp. 2541-2619, 2014.

[18] K. Kimura, T. Kimura, M. Ishihara et al., "JCS 2018 guideline on diagnosis and treatment of acute coronary syndrome," Circulation Journal, vol. 83, no. 5, pp. 1085-1196, 2019.

[19] L. Hongxu, W. Yongjian, and W. Xian, "Percutaneous coronary intervention in the treatment of perioperative myocardial injury expert's consensus," Chinese Journal of Integrative Medicine, vol. 4, pp. 6-10, 2017.

[20] M. S. Cetin, E. H. Ozcan Cetin, E. Kalender et al., "Monocyte to HDL cholesterol ratio predicts coronary artery disease severity and future major cardiovascular adverse events in acute coronary syndrome," Heart, Lung and Circulation, vol. 25, no. 11, pp. 1077-1086, 2016.

[21] M. J. Yuan, Y. S. Pan, and W. G. Hu, "A pilot study of prognostic value of non-invasive cardiac parameters for major adverse cardiac events in patients with acute coronary syndrome treated with percutaneous coronary intervention," International Journal of Clinical and Experimental Medicine, vol. 8, no. 12, pp. 22440-22449, 2015.

[22] World federation of Chinese Medicine Societies Interventional Cardiology Committee, "Percutaneous coronary intervention in the treatment of perioperative myocardial injury expert's consensus," Chinese Journal of Integrative Medicine, vol. 37, no. 4, pp. 389-393, 2017.

[23] D. Mother, A. Liberati, and J. Tetzlaff, "Preferred reporting items for systematic reviews and meta-analysis: the PRISMA statement," BMJ, vol. 339, p. 2535, 2009.

[24] J. Higgins, S. Green, and C. Collaboration, "Cochrane handbook for systematic reviews for interventions," Cochrane Database of Systematic Reviews, vol. 2, p. S38, 2011.

[25] B. Ibanez, S. James, and S. Agewall, "2017 ESC guidelines for the management of acute myocardial infarction in patients presenting with ST-segment elevation: the task force for the management of acute myocardial infarction in patients presenting with ST-segment elevation of the European society of cardiology (ESC)," European Heart Journal, vol. 26, no. 9, pp. 417-421, 2018.

[26] M. Roffi, C. Patrono, J.-P. Collet et al., "2015 ESC Guidelines for the management of acute coronary syndromes in patients presenting without persistent ST-segment elevation," European Heart Journal, vol. 37, no. 3, pp. 267-315, 2016.

[27] L. Aimin, "Clinical observation of different doses of tirofiban combined with heparin in the treatment of acute myocardial infarction," Journal of Chinese Practical Diagnosis and Therapy, vol. 27, no. 5, pp. 485-487, 2013.

[28] K. A. Hicks, J. E. Tcheng, and B. Bozkurt, "2014 ACC/AHA key data elements and definitions for cardiovascular endpoint events in clinical trials," Journal of Nuclear Cardiology, vol. 22, no. 5, pp. 1041-1144, 2015. 
[29] S. J. Baron, Y. Lei, K. Chinnakondepalli et al., "Economic outcomes of bioresorbable vascular scaffolds versus everolimus-eluting stents in patients undergoing percutaneous coronary intervention," JACC: Cardiovascular Interventions, vol. 10, no. 8, pp. 774-782, 2017.

[30] D. Bohning, U. Malzahn, and E. Dietz, "Some general points in estimating heterogeneity variance with the DerSimonianLaird estimator," Biostatistics, vol. 3, no. 4, pp. 445-457, 2002.

[31] A. Donald and A. Donner, "Adjustments to the MantelHaenszel chi-square statistic and odds ratio variance estimator when the data are clustered," Statistics in Medicine, vol. 6, no. 4, pp. 491-499, 1987.

[32] J. A. C. Sterne and M. Egger, "Funnel plots for detecting bias in meta-analysis," Journal of Clinical Epidemiology, vol. 54, no. 10, pp. 1046-1055, 2001.

[33] M. Egger, G. D. Smith, M. Schneider, and C. Minder, "Bias in meta-analysis detected by a simple, graphical test," $B M J$, vol. 315, no. 7109, pp. 629-634, 1997.

[34] J. Hongtao and L. Yuan, "Effects of Dazhuhongjingtian injection on the inflammatory factor and left ventricular function in the treatment of acute myocardial infarction patients after undergoing PCI," World Chinese Medicine, vol. 13, no. 3, pp. 635-639, 2018.

[35] L. Jia and H. Jun, "Clinical study on the protective effect and mechanism of Dazhuhongingtian injection on myocardial ischemia-reperfusion injury," Pharmacology and Clinics of Chinese Materia Medical, vol. 32, no. 1, pp. 175-178, 2016.

[36] S. Huirong, W. Dewei, and Z. Dandan, "Influence of Dazhuhongjingtian injection on serum MCP-1 and hsCRP in patients with unstable angina pectoris after percutaneous coronary intervention," Journal of Hebei Medical University, vol. 36, no. 10, pp. 1125-1128, 2015.

[37] C. Yushan, X. Jinhon, and G. Huaimin, "Effect of the Dazhuhongjingtian injection on inflammation reaction, fibrinolytic function and blood lipid in patients with acute myocardial infarction undergoing reperfusion therapy," Chinese Journal of Cardiovascular Research, vol. 12, no. 11, pp. 975-978, 2014.

[38] L. Xin, "Preventive and curative effect of Dazhuhongjingtian injection on myocardial ischemia reperfusion," Modern Journal of Integrated Traditional Chinese and Western Medicine, vol. 21, no. 28, pp. 3093-3094, 2012.

[39] W. Lanrong, "Clinical effect of Shuxuetong combined with Shenmai injection in the treatment of myocardial reperfusion injury," International Journal of Cardiovascular Diseases, vol. 44, no. 1, p. 171, 2017.

[40] S. Fengmei, Z. Haoliang, and C. Hui, "Effect of Shenmai injection on plasma apelin-13 and nitric oxide levels in patients with acute myocardial infarction," Zhejiang Journal of Traditional Chinese Medicine, vol. 52, no. 4, pp. 249-250, 2017.

[41] R. Lin, J. Xiaoxia, and L. Jia, "Effect of Shenmai injection on serum adhesion molecule and C-reactive protein in patients with acute coronary syndrome," Clinical Journal of Medical Officers, vol. 45, no. 3, pp. 295-299, 2017.

[42] Z. Zhaoxia, "Preventive effect of Shuxuetong combined with Shenmai injection on reperfusion injury in ASTEMI patients undergoing PCI," Practical Journal of Cardiac Cerebral Pneumal and Vascular Disease, vol. 25, no. 1, pp. 84-86, 2017.

[43] L. Lilan and T. Xiaoxiao, "Effect of Shenmai injection on patients with acute myocardial infarction before percutaneous coronary intervention," Journal of New Chinese Medicine, vol. 48, no. 10, pp. 24-26, 2016.
[44] W. Hua, L. Jingsong, and X. Shaoxin, "Effect of Shenmai injection on myocardial and microvascular protection in patients with acute myocardial infarction after percutaneous coronary intervention," Modern Journal of Integrated Traditional Chinese and Western Medicine, vol. 26, no. 11, pp. 1201-1203, 2017.

[45] L. Peng, L. Zhenqi, and Z. Huoqing, "Clinical observation on 30 cases of acute myocardial infarction treated by Shenmai injection combined with salvianate," Jiangsu Journal of Traditional Chinese Medicine, vol. 12, pp. 21-23, 2014.

[46] M. Caiyan, T. Hong, and L. Xiaoling, "Effects of combination of Shenmai and primary percutaneous coronary intervention on aldosterone, B-type natriuretic peptide and left ventricular function in patients with acute anterior myocardial infarction," Journal of Electrocardiology and Circulation, vol. 3, pp. 200-206, 2014.

[47] Y. Min, G. Hangyuan, and P. Fang, "Protective effects of Shenmai injection on myocardial ischemia reperfusion injury after emergency percutaneous coronary intervention," Chinese Journal of Integrated Traditional and Western Medicine in Intensive and Critical Care, vol. 18, no. 5, pp. 284-286, 2011.

[48] Y. Rong, C. Fenqiao, and C. Shaoyi, "Effect of Shenmai injection combined with ligustrazine injection on left ventricular remodelling after coronary intervention for acute myocardial infarction," Liaoning Journal of Traditional Chinese Medicine, vol. 35, no. 4, pp. 491-492, 2008.

[49] D. Fangming, X. Dexiang, and Z. Xinli, "Effect of compound Danshen injection combined with Shengmai powder on short-term prognosis of patients with myocardial infarction after PCI," Shandong Medical Journal, vol. 47, no. 27, pp. 150-151, 2007.

[50] W. Yonghao, W. Weihao, and L. Haiyun, "Protective effect of Danhong injection combined with creatine phosphate on myocardial function after PCI in patients with acute coronary syndrome," Journal of Cardiovascular Diseases of Integrated Chinese and Western Medicine, vol. 16, no. 21, pp. 60-63, 2018.

[51] Z. Guangwei, W. Chiyao, and X. Yugang, "Effect of Danhong injection on the serum IL-6 and IL-7 level in patients with acute myocardial infarction after percutaneous coronary intervention," International Journal of Pathology and Clinical Medicine, vol. 37, no. 9, pp. 1887-1893, 2017.

[52] L. Zhiqiang, L. Yibo, and Z. Lipei, "Observation on the efficacy of emergency PCI combined with Danhong injection in the treatment of non-ST-segment elevation acute myocardial infarction," Shaanxi Journal of Traditional Chinese Medicine, vol. 38, no. 7, pp. 869-870, 2017.

[53] Z. Weiwei, D. Hongwei, and Z. Wenquan, "Effects of Danhong injection on vascular endothelial function and inflammatory factors in elderly patients with acute coronary syndrome after coronary intervention," Chinese Journal of Gerontology, vol. 36, no. 22, pp. 5591-5593, 2016.

[54] H. Mengzhao, "Clinical study of intravenous injection of Danhong Injection combined with nitro-glycerine before PCI in patients with acute myocardial infarction," China Medical Herald, vol. 13, no. 24, pp. 152-155, 2016.

[55] L. Yang, X. Jinpeng, and D. Weiying, "Effect of Danhong injection on endothelial injury, degree of inflammation and cardiac function of patients with acute coronary syndrome after interventional therapy," Journal of Hainan Medical University, vol. 22, no. 15, pp. 1619-1622, 2016.

[56] J. Min, L. Junli, and G. Fang, "Effect of Danhong injection combined with Nitroglycerin on no-reflow and CRP in PCI 
treatment for AMI patients," Journal of Hebei Traditional Chinese Medicine and Pharmacology, vol. 1, pp. 5-7, 2015.

[57] X. Xinmin, C. Haiyang, and Y. Jing, "Protective effect of Danhong injection on the ischemic myocardium after percutaneous coronary intervention for acute myocardial infarction," Military Medical Journal of Southeast China, vol. 5, pp. 451-454, 2015.

[58] G. Jianfeng, W. Shengben, and Y. Jinping, "Treatment of Danhong injection with atorvastatin on endothelial function and inflammation factors in patients with acute coronary syndrome after percutaneous coronary intervention," Chinese Journal of Experimental Traditional Medical Formulae, vol. 21, no. 12, pp. 154-157, 2015.

[59] C. Yinghua and W. Lin, "Effect of Danhong injection on oxidative stress and inflammation reaction in patients with acute myocardial infarction undergoing percutaneous coronary intervention," Chinese Journal of Hospital Pharmacy, vol. 34, no. 3, pp. 215-218, 2014.

[60] Z. Yongxiang and Z. Qiang, "Effects of Danhong injection on protect myocardium of acute coronary syndrome patients after selective percutaneous coronary artery intervention," Chinese Journal of Experimental Traditional Medical Formulae, vol. 18, no. 23, pp. 308-310, 2012.

[61] H. Xiaonan, S. Tingting, and Z. Cheng, "Protective effect s of Danhong injection on myocardium after reperfusion," Liaoning Journal of Traditional Chinese Medicine, vol. 38, no. 8, pp. 1578-1580, 2011.

[62] Z. Beixin and Z. Shan, "Effect of Danhong injection on ET-1, sP-sel, and hs-CRP in patients with acute coronary syndrome undergoing percutaneous coronary intervention," Chinese Journal of Integrated Traditional and Western Medicine, vol. 31, no. 1, pp. 11-14, 2011.

[63] C. Hong, Z. Lixuan, and M. Xiaoning, "Protective effects of Danhong injection on myocardium after reperfusion in patients with acute myocardium infarction," Clinical Focus, vol. 25, no. 7, pp. 563-566, 2010.

[64] H. Yong, T. Zhiyan, and H. Dajun, "The effect of Danhong injection on platelet activation in patients with acute coronary syndrome after intervention," Chinese Journal of Modern Drug Application, vol. 2, no. 13, pp. 43-44, 2008.

[65] W. Zhihui, L. Zhibo, and M. Lihua, "The clinical observation of Danhong injection for the treatment of myocardial infarction after the intervention," China Practical Medical, vol. 4, no. 7, pp. 46-47, 2009.

[66] G. Xiaodong, Z. Pingshan, and K. Weiwei, "Clinical effect of emergency intervention combined with Danhong injection in the treatment of acute myocardial infarction," Practical Journal of Cardiac Cerebral Pneumal and Vascular Disease, vol. 16, no. 6, pp. 28-29, 2008.

[67] F. Kai, J. Xiaobo, and Q. Weiwei, "The effect of Danhong injection on cardiovascular event in earlier period and inflammatory reaction of the patients of ACS with PCI," Journal of Chinese Microcirculation, vol. 6, pp. 390-392, 2007.

[68] Y. Fan and L. Shayi, "Clinical observation of Breviscapine before percutaneous coronary intervention," Journal of Chinese Practical Diagnosis and Therapy, vol. 22, no. 11, pp. 823-824, 2008.

[69] S. Yuting and Y. Zheng, "Extracts of Gualoupi for patients with acute coronary syndrome effects of vascular endothelial and platelet function after PCI," Journal of Cardiovascular Diseases of Integrated Chinese and Western Medicine, vol. 15, no. 18, pp. 2237-2238, 2017.
[70] L. Hong, Y. Hua, and W. Lizhong, "Effect of Guanxinning injection on left ventricular remodelling in patients with acute ST-elevation myocardial infarction accompanied with diabetes mellitus undergoing percutaneous coronary intervention," Chinese General Practice, vol. 12, no. 23, pp. 2121-2123, 2009.

[71] L. Hong, Y. Hua, and W. Lizhong, "Application of Guanxinning injection after PCI in STEMI patient's results observation," Shandong Medical Journal, vol. 49, no. 34, p. 100, 2009.

[72] W. Rui, S. Meng, and Z. Nan, "Effects of safflower yellow on incidence of myonecrosis in patients with unstable angina after percutaneous coronary intervention," Chinese Journal of Integrative Medicine on Cardio/Cerebrovascular Disease, vol. 15, no. 10, pp. 1202-1204, 2017.

[73] Z. Weiwei, Z. Ying, and Z. Huawei, "Perioperative effect of Danhong Injection in patients with unstable angina pectoris during percutaneous coronary intervention," Chinese Journal of Evidence-Bases Cardiovascular Medicine, vol. 3, pp. 336-338, 2015.

[74] D. Chuntao and D. Lihua, "Protective effect with vascular endothelium of Danhong injection on unstable angina after coronary intervention in patients," Chinese Journal of Primary Medicine and Pharmacy, vol. 22, pp. 3407-3409, 2015.

[75] W. Yunshu, W. Fengde, and J. Longzhe, "The myocardial protective effect of safflower yellow in elderly patients with acute coronary syndrome after interventional therapy," Chinese Community Doctors, vol. 32, no. 21, pp. 104-105, 2016.

[76] Z. Dingxue and L. Wenbao, "Forty-four cases with acute coronary syndrome treated with tirofiban hydrochloride combined with Safflower injection," Henan Traditional Chinese Medicine, vol. 35, no. 9, pp. 2084-2086, 2015.

[77] J. Xian, H. Wei, and G. Jun, "Curative effect of Safflower injection on non-ST elevation acute coronary syndrome and protective effect on interventional therapy," Modern Journal of Integrated Traditional Chinese and Western Medicine, vol. 19, no. 14, pp. 1698-1699, 2010.

[78] L. Suyun, Z. Hui, and L. Yongjun, "The protective effect and mechanism of Safflower injection on myocardial ischemia in interventional treatment of coronary heart disease," Journal of Hebei Traditional Chinese Medicine, vol. 19, no. 3, pp. 29-31, 2004.

[79] W. Yujuan and A. Maiti, "Impact of Kudiezi injection on postoperative acute myocardial infarction patients treated by PCI," Practical Journal of Cardiac Cerebral Pneumal and Vascular Disease, vol. 25, no. 4, pp. 81-84, 2017.

[80] L. Yuefan, Z. Xinli, and D. Faming, "Effect of Shengmai injection on inflammatory factors in patients with unstable angina pectoris after PCI," Chinese Journal of Clinical Research, vol. 25, no. 1, pp. 25-26, 2012.

[81] H. Yinghui, "Shengmai injection intervention on efficacy in the early percutaneous coronary intervention in patients with acute coronary syndrome," Shaanxi Journal of Traditional Chinese Medicine, vol. 5, pp. 554-557, 2015.

[82] W. Xuan, W. Yang, and S. Bin, "Clinical study of prevention and treatment for reperfusion injury with Shengmai injection after acute myocardial infarction PCI," Modern Journal of Integrated Traditional Chinese and Western Medicine, vol. 19, no. 28, pp. 3553-3554, 2010.

[83] L. Zhe, L. Hui, and L. Guofeng, "Impact of Shuxuetong injection combined with Alprostadil on acute myocardial infarction patients undergoing PCI," Practical Journal of 
Cardiac Cerebral Pneumal and Vascular Disease, vol. 26, no. 4, pp. 67-70, 2018.

[84] M. Xiaoyan, "Clinical observation on the effect of Shuxuetong injection in treatment of elderly acute myocardial infarction after PCI," Journal of Hubei University of Chinese Medicine, vol. 20, no. 1, pp. 75-77, 2018.

[85] Z. Zhenda, C. Cailian, and D. Ruimin, "Effect of Shuxuetong injection on cardiac function of AMI patients without reflow after percutaneous coronary intervention," Chinese Journal of Integrative Medicine on Cardio/Cerebrovascular Disease, vol. 10, pp. 1193-1195, 2015.

[86] F. Xuguang and N. Rong, "Effects of Shuxuetong injection on slow flow and no reflow during coronary intervention," Chinese Journal of Integrative Medicine on Cardio/Cerebrovascular Disease, vol. 11, no. 12, pp. 1438-1439, 2013.

[87] R. Tiezhou and H. Jie, "Effect of Shuxuetong injection on angina pectoris after direct coronary intervention in acute myocardial infarction," Chinese Journal of Integrative Medicine on Cardio/Cerebrovascular Disease, vol. 11, no. 12, pp. 1440-1441, 2013.

[88] Y. Yushuang, W. Jinyi, and L. Ying, "Effect of Shuxuetong injection on plasma soluble cell adhesion molecule-1 level in patients with acute myocardial infarction after percutaneous coronary intervention," Chinese Journal of Gerontology, vol. 27, no. 14, pp. 1401-1402, 2007.

[89] S. Jingchun, H. Guoming, and D. Zhongru, "Effects of Shuxuetong on acute coronary syndrome patients after interventional therapy," Shaanxi Medical Journal, vol. 38, no. 4, pp. 439-441, 2009.

[90] M. Jianping, M. Liusong, and L. Qicai, "Xiangdan injection and nitroglycerin were used to treat 30 cases of acute myocardial infarction," Journal of Practical Traditional Chinese Internal Medicine, vol. 21, no. 6, pp. 82-83, 2007.

[91] L. Huajin, Q. Zengyong, and M. Jiangwei, "Clinical study on Xuesaitong combined with percutaneous coronary intervention in treatment of acute ST-segment elevation myocardial infarction," Chinese Journal of Cardiovascular Research, vol. 16, no. 8, pp. 749-752, 2018.

[92] W. Lianren, "Application value of Xuesaitong injection in patients with acute ST-segment elevation myocardial infarction undergoing interventional therapy," International Medicine and Health Guidance News, vol. 24, no. 11, pp. 1679-1682, 2018.

[93] X. Danzhen and Q. Lingfei, "Effects of atorvastatin combined with Xuesaitong injection on inflammatory factors after PCI in patients with myocardial infarction," Chinese Journal of Rural Medicine and Pharmacy, vol. 25, no. 12, pp. 39-40, 2018.

[94] Q. Zhili, G. Fengmin, and X. Biao, "Effect of injection Xuesaitong on inflammatory factors and matrix metalloproteinases in patients with acute myocardial infarction after PCI," Chinese Journal of Integrative Medicine on Cardio/Cerebrovascular Disease, vol. 14, no. 20, pp. 2394-2396, 2016.

[95] G. Lijun, Z. Chunhui, and Z. Meng, "Effect of intracoronary injection with Xuesaitong in treating post-PCI slow-reflow phenomenon in patients with ST-segment elevation myocardial infarction," Chinese Journal of Integrated Traditional and Western Medicine, vol. 30, no. 4, pp. 348-351, 2010.

[96] H. Zhongchun, X. Huiyu, and S. Shunhua, "Effect of Xueshuantong combined with low molecular Heparin on inflammatory factors and endothelial function in patients with acute coronary syndrome after PCI," Progress in Modern Biomedicine, vol. 17, no. 18, pp. 3540-3543, 2017.
[97] H. Yingxin, W. Yiming, and J. Meizhi, "Study on the effect of early rehabilitation exercise combined with Xueshuantong injection on postoperative rehabilitation of patients with AMI and proBNP," Journal of Liaoning University of Traditional Chinese Medicine, vol. 19, no. 6, pp. 103-106, 2017.

[98] W. Ni, "Effects of Xueshuetong injection on serum lipids inflammatory factors and endothelial function in patients with acute coronary syndrome after interventional therapy," Modern Journal of Integrated Traditional Chinese and Western Medicine, vol. 26, no. 7, pp. 728-730, 2017.

[99] S. Yiguang, S. Kang, and C. Liwei, "Effects of Xueshuetong injection on serum lipids inflammatory cytokines and endothelial function in patients with acute coronary syndrome after interventional therapy," Modern Journal of Integrated Traditional Chinese and Western Medicine, vol. 25, no. 22, pp. 2478-2480, 2016.

[100] Z. Caihong and L. Jiuxi, "Effects of Xueshuetong injection on serum lipids inflammatory cytokines and endothelial function in patients with acute coronary syndrome after interventional therapy," Chinese Journal of Experimental Traditional Medical Formulae, vol. 21, no. 11, pp. 169-173, 2015.

[101] G. Hongyu and Z. Lan, "Effect of Yiqifumai injection on cardiac function after PCI in patients with acute myocardial infarction," Modern Journal of Integrated Traditional Chinese and Western Medicine, vol. 27, no. 25, pp. 2826-2828, 2018.

[102] C. B. Begg, "A measure to aid in the interpretation of published clinical trials," Statistics in Medicine, vol. 4, no. 1, pp. 1-9, 1985.

[103] M. Angell, "Negative studies," New England Journal of Medicine, vol. 321, no. 7, pp. 464-466, 1989.

[104] K. Dickersin, Y. Min, and C. L. Meinert, "Factors influencing publication of research results. Follow-up of applications submitted to two institutional review boardsfluencing publication of research results: follow-up of applications submitted to two institutional review boards," JAMA: The Journal of the American Medical Association, vol. 267, no. 3, pp. 374-378, 1992.

[105] G. Shan, Y. Peng, and Z. Yan, "Systematic review and metaanalysis of efficacy and safety of traditional Chinese medicine injections combined with western medicine in treatment of coronary heart disease with blood stasis syndrome," Liaoning Journal of Traditional Chinese Medicine, vol. 45, no. 3, pp. 449-453, 2018.

[106] J. Zhaochen, H. Haiying, and Y. Fengwen, "Network metaanalysis of Yiqihuoxue Chinese patent medicine for coronary heart disease with angina pectoris," China Journal of Chinese Materia Medical, vol. 44, no. 9, pp. 1927-1937, 2019.

[107] W. Junyan, Z. Sicheng, and H. Xinyong, "Meta-analysis of efficacy on angina pectoris treated by supplementing $Q i$ and activating blood circulation, removing phlegm and blood stasis," Chinese Archives of Traditional Chinese Medicine, vol. 36, no. 11, pp. 2647-2650, 2018.

[108] L. Min, H. Li, and W. Weigang, "Myocardial protection of Chinese herbal medicine pharmacological postconditioning for patients with acute myocardial infarction: a meta-analysis," Liaoning Journal of Traditional Chinese Medicine, vol. 40, no. 2, pp. 240-245, 2013.

[109] Q. Weibin, H. Guixin, and C. Yalu, "Meta-analysis of efficacy of reinforcing $Q i$, replenishing Yin and promoting blood circulation combined with percutaneous coronary intervention to improve myocardial microcirculation," Liaoning Journal of Traditional Chinese Medicine, vol. 45, no. 5, pp. 900-904, 2018. 
[110] Y. Yu, E. S. Spatz, Q. Tian et al., "Traditional Chinese medicine use in the treatment of acute heart failure in western medicine hospitals in China: analysis from the China PEACE retrospective heart failure study," Journal of the American Heart Association, vol. 8, no. 15, Article ID e012776, 2019. 